\title{
RATIONAL APPROXIMATIONS TO IRRATIONAL COMPLEX
}

\section{NUMBERS*}

\author{
BY
}

LESTER R. FORD

We shall call a number $m+n i$, where $i=\sqrt{-1}$, a complex integer if $m$ and $n$ are real integers. By a rational complex fraction we shall mean a fraction whose numerator and denominator are complex integers. By an irrational complex number we shall mean a number not expressible as a rational complex fraction.

Hermite $\dagger$ has proposed a method of forming a suite of rational complex fractions approaching an irrational complex number $\omega$. The method is briefly the following. Consider the positive definite hermitian form

$$
F(x, y)=(x-\omega y)(\bar{x}-\bar{\omega} \bar{y})+k^{2} y \bar{y},
$$

where $\bar{x}, \bar{y}, \bar{\omega}$ are the conjugate imaginaries of $x, y, \omega$, and where $k$ is real. Let $x=p, y=q$ be a pair of complex integers giving a minimum value of the form $F$ (the values $x=0, y=0$ being excluded). Then the fraction $p / q$ is an approximation to $\omega$ satisfying the inequality

$$
\left|\omega-\frac{p}{q}\right| \leqq \frac{1}{\sqrt{2} q \vec{q}} .
$$

If $k$ be made to decrease from $\infty$ to 0 , the integers $p$ and $q$ change from time to time, and there results an infinite suite of fractions tending toward $\omega$. If $\omega$ be a rational complex fraction, a suite is likewise determined, but it terminates with the fraction $\omega$ after a finite number of terms.

It is the object of the first part of this paper to investigate the suite of Hermite, to give the fractions a geometrical interpretation, to find a method of calculating them, and to study their properties.f

The second part of the paper is devoted to approximations by means of

* Part II presented to the Society, Sept. 4, 1916, under the title Regular continued fractions.

† Sur la théorie des formes quadratiques, Journal für Mathematik, vol. 47 (1854), particularly pp. 357-366.

† The principal results of this part have been presented in a brief note in $\mathrm{Com} \mathrm{p} \mathrm{te} \mathrm{s}$ Ren d u s, vol. 162 (1916), pp. 459-461.

Trans. Am. Math. Soc. 1 
continued fractions. It is shown that the convergents of any continued fraction of the form

$$
s_{0} \pm \frac{\epsilon_{1} \mid}{\mid s_{1} \pm} \frac{\epsilon_{2} \mid}{\mid s_{2} \pm} \cdots \frac{\epsilon_{n} \mid}{\mid s_{n} \pm} \cdots,
$$

where $\epsilon_{n}= \pm 1$ or $\pm i$, and where $s_{n}$ is a complex integer (such a fraction will be called regular), can be derived by a method which is a generalization of the method of Hermite. The properties of the convergents of such a continued fraction, in particular their values as approximations to the sum of the fraction, are investigated, and theorems relative to the periodicity of the continued fraction are established.

I take occasion here to express my indebtedness to Professor Humbert of the Collège de France, who suggested to me a study of the suite of Hermite, and who pointed the way to its geometrical interpretation in his investigation of the analogous problem for the case of reals.*

\section{PART I \\ The fractions of Hermite}

1. The Group of Picard. The whole theory of rational approximations to complex numbers is concerned with the group of transformations

$$
z^{\prime}=\frac{\alpha z+\beta}{\gamma z+\delta} \quad(\alpha \delta-\beta \gamma=1)
$$

where $\alpha, \beta, \gamma, \delta$ are complex integers. This group is known as the Group of Picard. The group is not properly discontinuous in the z-plane, but if it be defined as a group of space transformations according to the idea of Poincaré $\dagger$ a fundamental polyhedron for the group exists. The space transformations, where $\zeta$ is a variable measured along an axis through the origin perpendicular to the $z$-plane, are

$$
\begin{aligned}
\rho^{\prime 2} & =\frac{\rho^{2} \alpha \bar{\alpha}+z \alpha \bar{\beta}+\bar{z} \bar{\alpha} \beta+\beta \bar{\beta}}{\rho^{2} \gamma \bar{\gamma}+z \gamma \bar{\delta}+\bar{z} \bar{\gamma} \delta+\delta \bar{\delta}}, \\
z^{\prime} & =\frac{\rho^{2} \alpha \bar{\gamma}+z \alpha \bar{\delta}+\bar{z} \beta \bar{\gamma}+\beta \bar{\delta}}{\rho^{2} \gamma \bar{\gamma}+z \gamma \bar{\delta}+\bar{z} \bar{\gamma} \delta+\delta \bar{\delta}}, \\
\bar{z}^{\prime} & =\frac{\rho^{2} \bar{\alpha} \gamma+z \gamma \bar{\beta}+\bar{z} \bar{\alpha} \delta+\bar{\beta} \delta}{\rho^{2} \gamma \bar{\gamma}+z \gamma \bar{\delta}+\bar{z} \bar{\gamma} \delta+\delta \bar{\delta}},
\end{aligned}
$$

where $\rho^{\prime 2}=\zeta^{\prime 2}+z^{\prime} \bar{z}^{\prime}$ and $\rho^{2}=\zeta^{2}+z \bar{z} . \S$ Combining these equations we

*Comptes Rendus, vol. 161 (1915), pp. 717-721; Journalde mathé $\mathrm{m}$ a t i q u e s, 7th ser., vol. 2 (1916), pp. 79-103.

$\dagger$ Mémoire sur les groupes kleinéens, A c t a M a t h e m a t i c a , vol. 3 (1884), pp. 49-92.

$\S$ Poincaré, loc. cit., p. 54. 
get also

$$
\zeta^{\prime}=\frac{\zeta}{\rho^{2} \gamma \bar{\gamma}+z \gamma \bar{\delta}+\bar{z} \bar{\gamma} \delta+\delta \bar{\delta}} .
$$

For points of the z-plane the transformation (5) is equivalent to (4).

The transformation inverse to (4) is

$$
z^{\prime}=\frac{-\delta z+\beta}{\gamma z-\alpha}
$$

hence the inverse space transformation can be written down by replacing $\alpha, \bar{\alpha}, \delta, \bar{\delta}$ by $-\delta,-\bar{\delta},-\alpha,-\bar{\alpha}$ respectively in (5) and (6).

These space transformations preserve angles both in magnitude and sign, transform spheres into spheres and circles into circles (planes and straight lines being included as special cases of spheres and circles), and transform figures inverse with respect to a sphere into figures inverse with respect to the transformed sphere. These properties are a consequence of the fact that each transformation is equivalent to an even number of inversions in spheres.

The fundarnental polyhedron for the Group of Picard has been found by Bianchi* to be the pentahedron lying above the sphere $\xi^{2}+\eta^{2}+\zeta^{2}=1$ $(z=\xi+i \eta)$ and enclosed by the four planes $\xi=0, \xi=\frac{1}{2}, \eta=-\frac{1}{2}, \eta=\frac{1}{2}$. The transforms of this region by the transformations of the group fill up, without overlapping, the whole space above the z-plane. This division of the half-space can also be derived from the fundamental pentahedron by inverting it in each of its faces, inverting each new pentahedron in each of its faces, and so on ad infinitum.

In what follows we shall find it convenient to adjoin to the fundamental pentahedron that arising from it by reflection in the plane $\xi=0$. This double pentahedron, which we shall henceforth call the fundamental pentahedron, then lies above the sphere $\xi^{2}+\eta^{2}+\zeta^{2}=1$ and is bounded by the planes $\xi= \pm \frac{1}{2}, \eta= \pm \frac{1}{2}$. The four plane faces we shall call the lateral faces of the pentahedron, and the remaining face we shall call its base. Of the five vertices, that at $\infty$ we shall call the peak, reserving for the other four the name vertices. The vertices lie in the plane $\zeta=\frac{1}{2} \sqrt{2}$.

If a transformation of the group be applied to this pentahedron there results a pentahedron bounded by spheres orthogonal to the z-plane, and we shall apply to the faces and vertices of this pentahedron the names of the corresponding parts in the fundamental pentahedron. Thus the four lateral faces meet in a point, the peak, in the $z$-plane. The four vertices and the base lie in the space above the $z$-plane.

The repetitions of the fundamental pentahedron by the translations of the group $z^{\prime}=z+\beta$ fill the whole space outside the spheres of unit radius

\footnotetext{
* Mathematis che Annalen, vol. 38 (1891), pp. 313-333.
} 
with centers at the points of the $z$-plane whose $z$-coördinates are complex integers. Each of these pentahedra has its peak at $\infty$ and its vertices in the plane $\zeta=\frac{1}{2} \sqrt{2}$.

The transformation $z^{\prime}=-1 / z$ carries the fundamental pentahedron into another with the same base and with its peak at the origin. By the transformations $z^{\prime}=z+\beta$ this pentahedron is carried into others with bases in common with those of the preceding paragraph and with peaks at the points of the z-plane whose coördinates are complex integers.

2. The positive definite hermitian form. The binary form

$$
\phi(x, y)=a x \bar{x}+b x \bar{y}+\bar{b} \bar{x} y+c y \bar{y},
$$

where $a$ and $c$ are real, is called a hermitian form. It is definite if the determinant, $D=b \bar{b}-a c$, is negative. We shall suppose that $a>0$; that is, that the form is positive. If we set $x / y=z$ and equate (8) to zero, we obtain the equation of an imaginary circle in the $z$-plane,

$$
a z \bar{z}+b z+\bar{b} \bar{z}+c=0 .
$$

The one-parameter family of spheres through this circie contains two real point spheres; these are, if we write $b=b_{1}+i b_{2}$, at the points

$$
\xi=-\frac{b_{1}}{a}, \quad \eta=\frac{b_{2}}{a}, \quad \zeta= \pm \frac{\sqrt{-D}}{a} .
$$

The point lying in the upper half space $(\zeta=+\sqrt{-D} / a)$ is customarily chosen as the representative point of the form.* This point has the property that if $x$ and $y$ be replaced by the new variables

$$
X=\alpha x+\beta y, \quad Y=\gamma x+\delta y \quad(\alpha \delta-\beta \gamma=m \neq 0)
$$

where $m$ is real, the representative point of the new form is derived from that of the old by the space transformation corresponding to

$$
Z=\frac{\alpha z+\beta}{\gamma z+\delta} .
$$

In the form (1) of the Introduction $D=-k^{2}$. Writing $\omega=\omega_{1}+i \omega_{2}$ we find the representative point of the form to be

or

$$
\xi=\omega_{1}, \quad \eta=\omega_{2}, \quad \zeta=+\sqrt{k^{2}},
$$

$$
z=\omega, \quad \zeta=k,
$$

where $k$ is the positive square root of $k^{2}$. As $k$ decreases from $\infty$ to 0 , the representative point traces the half of the line $z=\omega$ lying in the upper half space, passing from $\infty$ to the $z$-plane.

* Fricke-Klein, Theorie der automorphen Functionen, vol. 1, p. 455. 
Minimum of the form. As a step toward finding the minimum of the form (8) - that is, the minimum for complex integral values of $x$ and $y(x=0$, $y=0$ excluded)-we shall establish the following proposition.

If the representative point of the form lies in a pentahedron whose peak is at infinity the minimum of the form is the first coefficient.

The first coefficient is a permissible value, being given by $x=1, y=0$. Obviously $x=p, y=0$ where $|p|>1$ yields a greater value. We shall treat two cases: $x=p, y=1$, and $x=p, y=q$, where $|q|>1$. Thus all possible values of $x$ and $y$ are considered.

The representative point of the form lies outside the unit sphere whose center is $z=p$; that is, outside the sphere

$$
(z-p)(\bar{z}-\bar{p})+\zeta^{2}=1 .
$$

The coördinates of the representative point from (10) are

$$
z=\left(-b_{1}+i b_{2}\right) / a=-\bar{b} / a, \quad \zeta=\sqrt{a c-b \bar{b}} / a,
$$

and these then satisfy the inequality

whence

$$
\left(-\frac{\bar{b}}{a}-p\right)\left(-\frac{b}{a}-\bar{p}\right)+\frac{a c-b \bar{b}}{a^{2}} \geqq 1,
$$

$$
a p \bar{p}+b p+\bar{b} \bar{p}+c \geqq a .
$$

That is, $\phi(p, 1) \geqq a$, whatever the integer $p$ may be.

The representative point also lies above the plane $\zeta=\frac{1}{2} \sqrt{2}$. Then

But

$$
\frac{a c-b \bar{b}}{a^{2}} \geqq \frac{1}{2} \text {. }
$$

$$
\begin{aligned}
\phi(p, q) & =a p \bar{p}+b p \bar{q}+\bar{b} \bar{p} q+c q \bar{q} \\
& =a\left[\left(p+\frac{\bar{b} q}{a}\right)\left(\bar{p}+\frac{b \bar{q}}{a}\right)+\frac{a c-b \bar{b}}{a^{2}} q \bar{q}\right] \\
& \geqq \frac{a q \bar{q}}{2} \geqq a,
\end{aligned}
$$

since $q \bar{q} \geqq 2$. The proposition is thus established.

Suppose now that the representative point does not lie in a pentahedron whose peak is at $\infty$. We observe that the set of values of the form given by complex integral values of the variables is unchanged if we make the substitution

$$
x=\alpha X+\beta Y, \quad y=\gamma X+\delta Y, \quad \alpha \delta-\beta \gamma=1,
$$

$\alpha, \beta, \gamma, \delta$ being complex integers. For complex integral values of $X$ and $Y$ 
yield complex integral values of $x$ and $y$, and conversely. The new form in $X$ and $Y$ will have the same minimum as the original.

Now et

$$
z=\frac{\alpha Z+\beta}{\gamma Z+\delta}
$$

be the transformation of the Group of Picard carrying the fundamental pentahedron into the pentahedron in which the representative point of the form (8) lies; and let the coefficients $\alpha, \beta, \gamma, \delta$ of (15) be used in the substitution (14). Then the representative point of the new form lies within the fundamental pentahedron, and its minimum is the first coefficient. The new form we find, on making the substitution (14), to be

$$
\phi^{\prime}(X, Y)=(a \alpha \bar{\alpha}+b \alpha \bar{\gamma}+\bar{b} \bar{\alpha} \gamma+c \gamma \bar{\gamma}) X \bar{X}+\cdots
$$

The first coefficient is then $\phi(\alpha, \gamma)$.

The peak of the pentahedron in which the representative point of the form $\phi(x, y)$ lies is the transform of $Z=\infty$; that is, $z=\alpha / \gamma$. We have thus proved the first part of the following theorem:

THEOREM. If $\alpha / \gamma$ (in its lowest terms) is the z-coördinate of the pealc of the pentahedron in which the representative point of the form $\phi(x, y)$ lies, the minimum of the form is $\phi(\alpha, \gamma)$.

Conversely, if $\phi(\alpha, \gamma)$ is the minimum of the form the peak of the pentahedron in which its representative point lies has the z-coördinate $\alpha / \gamma$.

The converse follows at once in case the form attains its minimum for a single pair of variables. We see from the derivation of (12) and (13) that the minimum of the form is given by a single pair of values in general. Now (12) becomes an equality only if the representative point lies in a base common to two pentahedra and (13) becomes an equality if the representative point lies in a vertex, where several pentahedra meet. In these exceptional cases there are two or more pairs of values giving the minimum, but we easily show that this arises from the fact that the representative point may be said to belong to two or more pentahedra.

3. Geometrical interpretation of the fractions of Hermite. The representative point of the form (1) of the Introduction we found (11) to be $z=\omega$, $\zeta=k$. The fraction of Hermite for a given value of $k$ is $p / q$ where $x=p$, $y=q$ gives the minimum of the form; that is, it is the $z$-cöordinate of the peak of the pentahedron in which the representative point of the form lies. As $k$ decreases from $\infty$ to 0 the representative point traces the half-line $z=\omega$ from $\infty$ to the $z$-plane. We have then the following interpretation:

Let a moving point trace the half of the line $z=\omega$ lying in the upper half-space, passing from $\infty$ to the z-plane. The z-coördinates of the peaks of the successive pentahedra through which this point passes are the fractions of Hermite tending toward the value $\omega$. 
From this interpretation it is obvious that there are only a finite number of fractions if $\omega$ is rational. The moving point eventually enters and remains within pentahedra with peaks at $\omega$, and the suite terminates.

4. The $S$ - and $\Sigma$-spheres. In this section we shall deduce certain geometrical facts concerning the pentahedral division of the half-space which will be frequently used in the following pages.

A plane, $\zeta=h$, parallel to the $z$-plane is transformed by a transformation of the group, $z^{\prime}=(\alpha z+\beta) /(\gamma z+\delta)$, into a sphere tangent to the z-plane. We get its equation from the inverse of equation (6). It is

$$
h=\frac{\zeta}{\rho^{2} \gamma \bar{\gamma}-z \gamma \bar{\alpha}-\bar{z} \bar{\gamma} \alpha+\overline{\alpha \bar{\alpha}} .}
$$

Since $\rho^{2}=z \bar{z}+\zeta^{2}$, this can be written

$$
\left(z-\frac{\alpha}{\gamma}\right)\left(\bar{z}-\frac{\bar{\alpha}}{\bar{\gamma}}\right)+\left(\zeta-\frac{1}{2 h \gamma \bar{\gamma}}\right)^{2}=\frac{1}{4 h^{2} \gamma^{2} \bar{\gamma}^{2}} .
$$

From this we see that the center of the sphere is the point $z=\alpha / \gamma, \zeta=1 / 2 h \gamma \bar{\gamma}$, and that its radius is $1 / 2 h \gamma \bar{\gamma}$. The sphere is thus tangent to the $z$-plane at the point $z=\alpha / \gamma$.

There are two planes of particular importance of the form $\zeta=h$. One of these is $\zeta=\frac{1}{2} \sqrt{2}$, which contains the vertices of all pentahedra with peaks at $\infty$. By the transformation mentioned above this becomes a sphere of radius $1 / \sqrt{2} \gamma \bar{\gamma}$ tangent to the $z$-plane at $z=\alpha / \gamma$. We shall call this sphere $\Sigma(\alpha / \gamma)$. The transformation in question carries $z=\infty$ to $z^{\prime}=\alpha / \gamma$. The part of the plane above $\zeta=\frac{1}{2} \sqrt{2}$ is carried into the interior of $\Sigma(\alpha / \gamma)$. Hence all the pentahedra with peaks at $z=\alpha / \gamma$ lie within the sphere $\Sigma(\alpha / \gamma)$ and their vertices lie upon its surface.

A second important plane is $\zeta=1$, which is tangent to the bases of all the pentahedra with peaks at $\infty$. It is transformed into a sphere of radius $1 / 2 \gamma \bar{\gamma}$ tangent to the $z$-plane at $\alpha / \gamma$. This sphere we shall call $S(\alpha / \gamma)$. We see then that the bases of all pentahedra with peaks at $z=\alpha / \gamma$ are tangent to the sphere $S(\alpha / \gamma)$.

It is well known that if $p$ and $q$ are any two complex integers without a common factor, other two complex integers, $\beta$ and $\delta$, can be found such that $p \delta-\beta q=1$. Then $z^{\prime}=(p z+\beta) /(q z+\delta)$ is a transformation of the Group of Picard which carries $z=\infty$ to $z^{\prime}=p / q$. Hence, each rational point, $p / q$, of the z-plane is a peak for infinitely many pentahedra. The vertices of these pentahedra lie upon the surface of $\Sigma(p / q)$ and their bases are tangent to $S(p / q)$.

We shall find it convenient to represent the point $\infty$ by $1 / 0$. For the sake of uniformity we shall designate the planes $\zeta=1$ and $\zeta=\frac{1}{2} \sqrt{2}$ by 
$S(1 / 0)$ and $\Sigma(1 / 0)$ respectively. The space above these planes corresponds to the interiors of the other $S$ - or $\Sigma$-spheres.

Consider now $S(p / q)$. Its diameter is $1 / q \bar{q}$. The sphere $S(p / q)$ will lie below $\zeta=1$ in general, but will be tangent if $q \bar{q}=1$. In this latter case $p / q=s$, a complex integer, and we know that a pentahedron with peak at $s$ and another with peak at $\infty$ have a base in common. The point of tangency lies in the common base. Since $\zeta=1$ can be transformed into any other $S$ sphere we have the result:

The sphere $S\left(p^{\prime} / q^{\prime}\right)$ will be tangent to the sphere $S(p / q)$ if a pentahedron with peak at $p^{\prime} / q^{\prime}$ has a base in common with a pentahedron with peak at $p / q$; in all other cases the spheres are entirely exterior to one another.

The diameter of $\Sigma(p / q)$ is $\sqrt{2} / q \bar{q}$. If $q \bar{q}=1$, so that $p / q=s / 1$, the center is at $z=s, \zeta=1 / \sqrt{2}$. The center thus lies in the plane $\zeta=\frac{1}{2} \sqrt{2}$, and $\Sigma(s / 1)$ and $\Sigma(1 / 0)$ cut orthogonally. Again, if $q \bar{q}=2$ (that is, $q=$ $\pm 1 \pm i)$ the diameter of $\Sigma(p / q)$ is $\frac{1}{2} \sqrt{2}$, and $\Sigma(p / q)$ touches $\Sigma(1 / 0)$. By a simple division we can put $p / q$ in the form $s+1 /(1-i)$, or $s+$ $(1+i) / 2$, where $s$ is an integer. The point of tangency is $z=s+(1+i) / 2$, $\zeta=\frac{1}{2} \sqrt{2}$, a vertex of certain of the pentahedra with peaks at $\infty$. It follows that in this case certain pentahedra with peaks at $p / q$ must also have vertices at this point of tangency. If $q \bar{q}>2, \Sigma(p / q)$ lies entirely below $\zeta=\frac{1}{2} \sqrt{2}$. Since $\zeta=\frac{1}{2} \sqrt{2}$ can be transformed into any other $\Sigma$-sphere we have the result:

The sphere $\Sigma\left(p^{\prime} / q^{\prime}\right)$ will intersect the sphere $\Sigma(p / q)$ orthogonally if a pentahedron with peak at $p^{\prime} / q^{\prime}$ and a pentahedron with peak at $p / q$ have a common base; $\Sigma\left(p^{\prime} / q^{\prime}\right)$ will touch $\Sigma(p / q)$ if a pentahedron with peak at $p^{\prime} / q^{\prime}$ and a pentahedron with peak at $p / q$ have a common vertex but no common base; the two $\Sigma$-spheres will be entirely exterior to one another in all other cases.

The analytical condition for a common base is expressed in the following

THEOREM. The necessary and sufficient condition that a pentahedron with peak at $p^{\prime} / q^{\prime}$ and a pentahedron with peak at $p / q$ (the fractions being in their lowest terms) have a common base is that $p q^{\prime}-p^{\prime} q= \pm 1$ or $\pm i$.

If two such pentahedra have a common base, let $z^{\prime}=(\alpha z+\beta) /(\gamma z+\delta)$ be the transformation of the Group of Picard carrying the fundamental pentahedron into the one with peak at $p / q$. Then the pentahedron with peak at the origin and with the same base as the fundamental pentahedron is carried into the one with peak at $p^{\prime} / q^{\prime}$. But $z=\infty$ and $z=0$ become respectively $\alpha / \gamma$ and $\beta / \delta$, and the relation $\alpha \delta-\beta \gamma=1$ shows that $\alpha$ and $\gamma$, also $\beta$ and $\delta$, are without a common factor. Since $p / q=\alpha / \gamma, p^{\prime} / q^{\prime}=\beta / \delta$, we have $p=\epsilon \alpha, q=\epsilon \gamma, p^{\prime}=\eta \beta, q^{\prime}=\eta \delta$, where $\epsilon$ and $\eta$ are of the form \pm 1 or $\pm i$. Then

$$
p q^{\prime}-p^{\prime} q=\epsilon \eta(\alpha \delta-\beta \gamma)=\epsilon \eta= \pm 1 \quad \text { or } \quad \pm i .
$$

The condition is thus necessary. 
It is also sufficient. For if $p q^{\prime}-p^{\prime} q= \pm 1$ or $\pm i$ the transformation $z^{\prime}=\left(p z+\epsilon p^{\prime}\right) /\left(q z+\epsilon q^{\prime}\right)$, where $\epsilon= \pm 1$ or $\mp i$ respectively, has determinant 1 and is thus of the Group of Picard. It transforms $\infty$ and 0 into $p / q$ and $p^{\prime} / q^{\prime}$ respectively. Now a pentahedron with peak at $\infty$ and one with peak at 0 have a common base, and these are carried into two with a common base and with peaks at $p / q$ and $p^{\prime} / q^{\prime}$. The theorem is thus established.

5. The calculation of the fractions of Hermite. According to the interpretation (Section 3) the calculation of the fractions reduces to the problem of finding the z-coördinates of the successive peaks of the pentahedra through which the line $z=\omega$ passes. We observe that two pentahedra with a common lateral face have the same peak; hence a moving point on the line $z=\omega$ can pass from one pentahedron into another with a different peak only by passing through the common base of the two pentahedra.* An application of the theorem at the close of the preceding section gives the following result:

If $p^{\prime} / q^{\prime}$ and $p / q$ are successive fractions of Hermite in the development of a number $\omega, p q^{\prime}-p^{\prime} q= \pm 1$ or $\pm i$. $\dagger$

By multiplying $p$ and $q$ by a suitable one of the numbers $\pm 1, \pm i$, we can have $p q^{\prime}-p^{\prime} q$ equal always to any one of the four quantities desired. The method of calculation to be given yields always $p q^{\prime}-p^{\prime} q=-1$, where $p / q$ is the fraction following $p^{\prime} / q^{\prime}$.

The point $z=\omega, \zeta=k$ lies in a pentahedron with peak at $\infty$ if $k$ is large. The first fraction is then $1 / 0$, whatever the value of $\omega$. The pentahedra with bases in common with those with peaks at $\infty$ have their peaks at the complex integers, and the common bases project orthogonally upon the $z$-plane into unit squares whose sides are parallel to the coördinate axes and whose centers are the integers. The second peak is then at a, the nearest complex integer to $\omega$; that is, an integer such that the real part and the imaginary part of $\omega-a$ are each less than or equal to $\frac{1}{2}$ in absolute value. Writing the second fraction $a / 1$ we have $a \times 0-1 \times 1=-1$.

We set now the following problem:

Given two successive fractions of Hermite, $p^{\prime} / q^{\prime}$ and $p / q$, in the development of a number $\omega$, where $p q^{\prime}-p^{\prime} q=-1$, to find the following fraction, $P / Q$.

The geometrical interpretation of the problem is shown in Figure 1, p. 10.

* If the line passes through a vertex of the first pentahedron the moving point may pass into a pentahedron having only a vertex in common with the first. But the vertex belongs also to other pentahedra, one of which has a base in common with the two preceding and can be taken as intermediate between them.

This case does not arise if $\omega$ is irrational, the $z$-coördinate of a vertex being always rational. For the vertex is the transform of one of the vertices of the fundamental pentahedron, $z=( \pm 1 \pm i) / 2, \zeta=\frac{1}{2} \sqrt{2}$, and we see from the second of equations (5) that with these values of $z$ and $\zeta, z^{\prime}$ is rational.

$\dagger$ This result appears in Hermite's memoir. 
As $k$ decreases the moving point in the line $z=\omega$ passes from a pentahedron $D_{1}$ with peak at $p^{\prime} / q^{\prime}$, traversing its base, and entering a pentahedron $D_{2}$ with

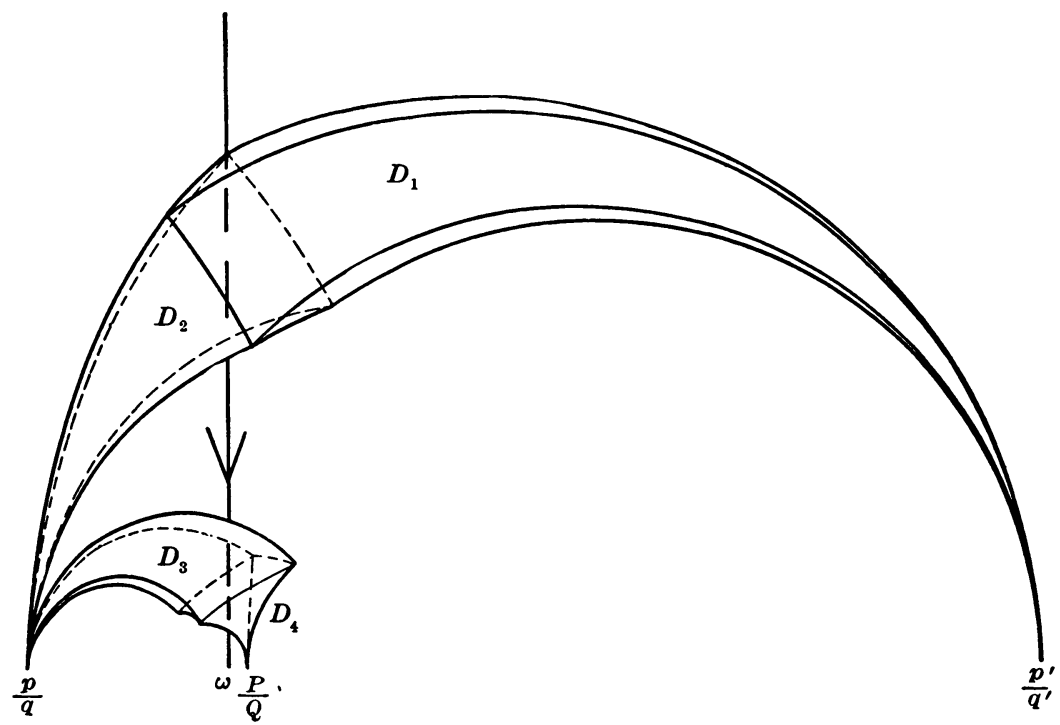

FIG. 1

peak at $p / q$. After passing through a certain number of pentahedra with peaks at $p / q$ it passes from one of them, $D_{3}$, by its base and enters a pentahedron, $D_{4}$, with its peak at $P / Q$, the fraction sought.

Let us apply to this configuration the transformation

$$
Z=\frac{q^{\prime} z-p^{\prime}}{q z-p},
$$

which, since its determinant is 1 , is of the Group of Picard. The transformed configuration is shown in Figure 2, $D_{1}^{\prime}, D_{2}^{\prime}, D_{3}^{\prime}, D_{4}^{\prime}$ being the transforms of

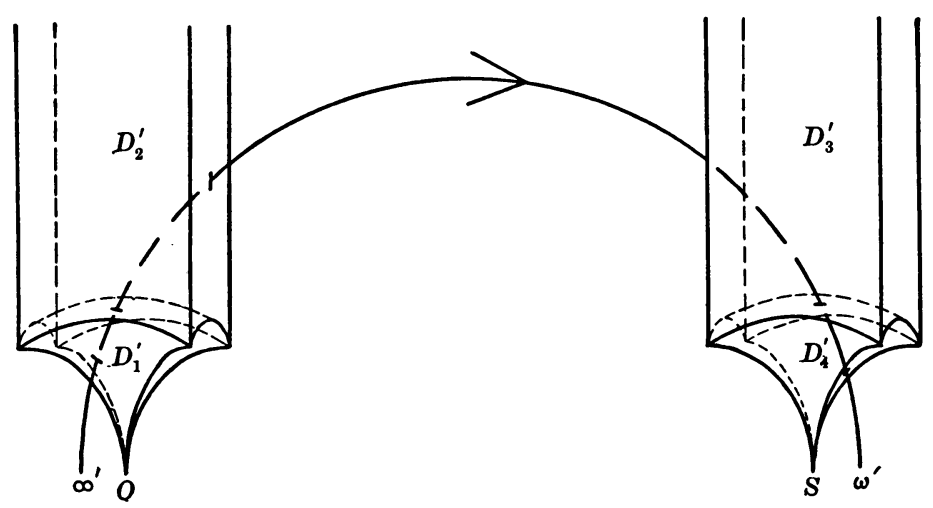

FIG. 2 
$D_{1}, D_{2}, D_{3}, D_{4}$. The fractions $p / q$ and $p^{\prime} / q^{\prime}$ become respectively $\infty$ and 0 . The pentahedron $D_{2}^{\prime}$ is then the fundamental pentahedron, and $D_{1}^{\prime}$ is the pentahedron with the same base. The pentahedron $D_{3}^{\prime}$ also has its peak at $\infty$, and $D_{4}^{\prime}$, which has the same base, has its peak at a complex integer $s$, to be found.

Writing $\infty^{\prime}$ and $\omega^{\prime}$ for the transforms of $\infty$ and $\omega$, we have

$$
\infty^{\prime}=\frac{q^{\prime}}{q}, \quad \omega^{\prime}=\frac{q^{\prime} \omega-p^{\prime}}{q \omega-p}=\frac{q^{\prime}}{q}-\frac{1}{u},
$$

where $u=q(q \omega-p)$. The half-line $z=\omega$ is transformed into the semicircle $C$ orthogonal to the $z$-plane through these two points. (This follows from the fact that circles are transformed into circles and that angles are preserved.) In tracing this semicircle from $\infty^{\prime}$ to $\omega$ we enter the pentahedra with peaks at $\infty$ by the base of $D_{2}^{\prime}$ and leave them by the base of $D_{3}^{\prime}$.

The circle $C$ has its center in the $z$-plane at $z=\frac{1}{2}\left(\infty^{\prime}+\omega^{\prime}\right)=q^{\prime} / q-1 / 2 u$, and its radius is $\frac{1}{2}\left|\infty^{\prime}-\omega^{\prime}\right|$, or $1 /|2 u|$. Its equations can be written

$$
\begin{aligned}
\left(z-\frac{q^{\prime}}{q}+\frac{1}{2 u}\right)\left(\bar{z}-\frac{\bar{q}^{\prime}}{\bar{q}}+\frac{1}{2 \bar{u}}\right)+\zeta^{2} & =\frac{1}{4 u \bar{u}}, \\
z-\frac{q^{\prime}}{q}+\frac{1}{2 u} & =\frac{t}{u},
\end{aligned}
$$

where $t$ is a real parameter. The first of these equations is the sphere with the same center and radius as $C$; the second is the plane through $\infty^{\prime}$ and $\omega^{\prime}$ orthogonal to the $z$-plane. The parameter $t$ is positive for points between $\infty^{\prime}$ and the highest point of $C$ and negative for points between the highest point and $\omega^{\prime}$.

The base common to $D_{3}^{\prime}$ and $D_{4}^{\prime}$ is the unit sphere with center at the point $z=s$ in the $z$-plane,

$$
(z-s)(\bar{z}-\bar{s})+\zeta^{2}=1 .
$$

The z-coördinate of the point of intersection of the circle (20) with the sphere (21) we find after an easy calculation to be

$$
z_{i}=\frac{q^{\prime}}{q}-\frac{1}{2 u}+\frac{1}{u}\left\{\frac{-\left(\frac{q^{\prime}}{q}-\frac{1}{2 u}-s\right)\left(\frac{\bar{q}^{\prime}}{\bar{q}}-\frac{1}{2 \bar{u}}-\bar{s}\right)-\frac{1}{4 u \bar{u}}+1}{\frac{1}{u}\left(\frac{\bar{q}^{\prime}}{\bar{q}}-\frac{1}{2 \bar{u}}-\bar{s}\right)+\frac{1}{\bar{u}}\left(\frac{q^{\prime}}{q}-\frac{1}{2 u}-s\right)}\right\}
$$

In order that the point of intersection lie in the base of $D_{3}^{\prime}, z_{i}$ must lie within the unit square whose center is $s$ and whose sides are parallel to the coördinate axes. This condition is also sufficient to determine $s$ if we rule out the case $s=0$, which is the peak of $D_{1}^{\prime}$. Otherwise stated, $s$ is the complex integer 
different from zero such that $z_{i}-s$ has a real and an imaginary part each less than or equal to $\frac{1}{2}$ in absolute value. Putting $U=q^{\prime} / q-1 / 2 u$, we can write $z_{i}-s$ in the following form

$$
U-s-\frac{u \bar{u}(U-s)(\bar{U}-\bar{s})-u \bar{u}+\frac{1}{4}}{u(u U+\bar{u} \bar{U})} .
$$

Having found $s$ to satisfy the above condition we can transform back to the original configuration by means of (18), i. e., $z=\left(p Z-p^{\prime}\right) /\left(q Z-q^{\prime}\right)$. Hence we have

and we can take

$$
\frac{P}{Q}=\frac{p s-p^{\prime}}{q s-q^{\prime}}
$$

$$
P=p s-p^{\prime}, \quad Q=q s-q^{\prime} .
$$

We note also that $P q-p Q=-1$.

The determination of $s$ from the expression (23) is not practicable, and a method will now be devised in which it will be necessary to have recourse to (23) only in certain doubtful cases.

The z-coördinate of the point of intersection of the semicircle $C$ with the plane $\zeta=\frac{1}{2} \sqrt{2}$ (which passes through the vertices of the pentahedra with peaks at $\infty$ ) will differ but little from that of the intersection with the base sought. Combining the equations of (20) and putting $\zeta^{2}=\frac{1}{2}$ we have

whence

$$
\frac{t^{2}}{u \bar{u}}+\frac{1}{2}=\frac{1}{4 u \bar{u}}
$$

$$
t= \pm \frac{1}{2} \sqrt{1-2 u \bar{u}} .
$$

The negative value of $t$ gives the intersection nearest $\omega^{\prime}$. From the second of equations (20) we have for $z_{0}$, the intersection sought,

$$
z_{0}=\frac{q^{\prime}}{q}-\frac{1}{2 u}(1+\sqrt{1-2 u \bar{u}}) .
$$

Let $s_{0}$ be the integer nearest $z_{0}$. Under what conditions is $s_{0}$ the integer $s$ of condition (23)? The semicircle $C$ either intersects the base $B$ lying above $z_{0}$, or intersects an adjoining base and passes under one of the sides bounding the base before reaching the plane $\zeta=\frac{1}{2} \sqrt{2} .^{*}$ Hence $s$ is one of the integers $s_{0}, s_{1}, s_{2}$, where $s_{1}$ and $s_{2}$ are the peaks of two of the pentahedra with bases adjoining $B$. Since $C$ passes through the base above the origin before arriving at the bases just mentioned, $\left|s_{1}\right|$ and $\left|s_{2}\right|$ are less than $\left|s_{0}\right|$. Also $\left|s_{1}-s_{0}\right|=1$ and $\left|s_{2}-s_{0}\right|=1$, since the bases above $s_{1}$ and $s_{2}$ adjoin $B$. (That is, one has

* This latter possibility renders the calculation of the fractions a more complicated procedure than in the case of reals. 
a real part, the other an imaginary part, differing by one unit from that of $s_{0}$.) Now the semicircle $C$ can intersect each sphere with center in the z-plane but once. Hence if $C$ intersects the base above $s_{1}$, say, the later point of intersection of $C$ with the plane $\zeta=\frac{1}{2} \sqrt{2}$ must still be within the unit sphere with center $s_{1}$, which forms that base. We find easily that $\zeta=\frac{1}{2} \sqrt{2}$ intersects this sphere in a circle of radius $\frac{1}{2} \sqrt{2}$. Hence if $\left(z_{0}-s_{1}\right)\left(\bar{z}_{0}-\bar{s}_{1}\right) \geqq \frac{1}{2}, C$ cannot have intersected the base above $s_{1}$.

We can now state the following rule of calculation, which would appear to be in as convenient a form as possible for actual use.

Let $s_{0}$ be the nearest complex integer to the number $z_{0}$, where

$$
z_{0}=\frac{q^{\prime}}{q}-\frac{1}{2 u}(1+\sqrt{1-2 u \bar{u}}) .
$$

Determine the complex integer $s$ as follows. Let $s_{1}, s_{2}$ be the two (in general two) complex integers such that

$$
\left|s_{0}-s_{i}\right|=1, \quad\left|s_{i}\right|<\left|s_{0}\right| \quad(i=1,2) .
$$

If neither of the inequalities

$$
\left(z_{0}-s_{i}\right)\left(\bar{z}_{0}-\bar{s}_{i}\right)<\frac{1}{2} \quad(i=1,2),
$$

is satisfied, $s=s_{0}$.

If the inequality is satisfied for $i=1$ (or. 2$),{ }^{*}$ substitute $s_{0}$ into the expression (23). If this expression has a real and an imaginary part each less than or equal to $\frac{1}{2}$ in absolute value, $s=s_{0}$; if not, $s=s_{1}\left(\right.$ or $\left.s_{2}\right)$.

Then

$$
P=p s-p^{\prime}, \quad Q=q s-q^{\prime} .
$$

Beginning with the two fractions $1 / 0$ and $a / 1$ and applying this method of procedure, we can calculate step by step the fractions of the suite.

In applying this process it is not necessary in the majority of cases to have recourse to condition (23). If such recourse is necessary there is recompense sometimes in the fact that $P_{1} / Q_{1}$, the fraction following $P / Q$, can be found at once. For if $s=s_{j}(j=1$ or 2$)$ in the preceding rule, we know that the next peak determined by $C$ is at $s_{0}$, for the point of intersection of $C$ with the plane $\zeta=\frac{1}{2} \sqrt{2}$ certainly lies in a pentahedron with peak at $s_{0}$. Therefore, if $P / Q=\left(p s_{i}-p^{\prime}\right) /\left(q s_{i}-q^{\prime}\right)$, we have $P_{1} / Q_{1}=\left(p s_{0}-p^{\prime}\right) /\left(q s_{0}-q^{\prime}\right)$. This yields the result

$$
P_{1}=\frac{p s_{0}-p^{\prime}}{s_{i}-s_{0}}, \quad Q_{1}=\frac{q s_{0}-q^{\prime}}{s_{i}-s_{0}},
$$

the denominators, which are of the form $\pm 1, \pm i$, being introduced in order to make $P_{1} Q-P Q_{1}=-1$.

\footnotetext{
*The inequality cannot be satisfied for both $s_{1}$ and $s_{2}$, since $\left|s_{1}-s_{2}\right|=\sqrt{2}$.
} 
6. The modified hermitian suite. The preceding rule of calculation gives all of the fractions of Hermite in the development of a number $\omega$. We get a suite of fractions differing little from the true suite if in the preceding process we take always $s=s_{0}$. We thus dispense altogether with condition (23), and find $P$ and $Q$ directly from (27) and (30). As we have just seen this usually gives the fraction immediately following $p / q$, but in some cases it yields the second fraction after $p / q$. This modified suite thus differs from the original by the omission of certain fractions, but no two successive fractions of the original suite are omitted.

It is easily seen that a fraction can be omitted only if the line $z=\omega$ passes through but two pentahedra with peaks at the fraction, but that it is not always omitted under these circumstances.

7. Properties of the fractions of Hermite. Let $p / q$ be a fraction in the suite determined by the number $\omega$. Then the line $z=\omega$ intersects pentahedra with peaks at $p / q$. As these pentahedra all lie within the sphere $\Sigma(p / q)$, which has the radius $1 / \sqrt{2} q \bar{q}$, we have at once the inequality (2) of the Introduction*

$$
\left|\omega-\frac{p}{q}\right| \leqq \frac{1}{\sqrt{2} q \bar{q}} .
$$

Since $p / q$ is the minimum of the form (1) for a certain value of $k$ the representative point of the form, $z=\omega, \zeta=k$, lies in a pentahedron with peak at $p / q$, and therefore lies in $\Sigma(p / q)$. This gives the condition

or

$$
\left(\omega-\frac{p}{q}\right)\left(\bar{\omega}-\frac{\bar{p}}{\bar{q}}\right)+\left(k-\frac{1}{\sqrt{2} q \bar{q}}\right)^{2} \leqq\left(\frac{1}{\sqrt{2} q \bar{q}}\right)^{2},
$$

$$
(p-\omega q)(\bar{p}-\bar{\omega} \bar{q})+k^{2} q \bar{q} \leqq k \sqrt{2} .
$$

This is the inequality from which Hermite, in the memoir cited, derives his results, and we thus see its geometrical meaning.

We turn now to a comparison of $p / q$ with the fraction $P / Q$ which immediately follows it in the suite.

Comparison of $Q$ and $q$. Let $M$ be the sphere forming the common base of the two pentahedra with peaks at $p / q$ and $P / Q$. The line $z=\omega$, traced from $\infty$ to the $z$-plane, enters this sphere through the common base, passing out of a pentahedron with peak at $p / q$ and entering one with peak at $P / Q$. The point $p / q$ of the $z$-plane is outside $M$ while $P / Q$ and also $\omega$ are inside. Now let us consider $S(p / q)$ and $S(P / Q)$, which are tangent to the bases of all pentahedra with peaks at $p / q$ and $P / Q$ respectively. Since the one

* If $\omega$ is irrational the sign of equality can be removed. For the only points of the pentahedra on the equator of $\Sigma(p / q)$ are vertices, and these have rational $z$-coördinates. A similar remark is applicable to the next inequality. See Note, Section 5. 
set of pentahedra can be derived from the other by an inversion in the common base, it follows that $S(p / q)$ and $S(P / Q)$ are inverse spheres with respect to $M$. The former lies outside $M$ and the latter inside, and they are tangent at a point in the common base. The former sphere is therefore larger than the latter. Using the values of the radii found in Section 4, we have $1 / 2 q \bar{q}$ $>1 / 2 Q \bar{Q}$; or

$$
|Q|>|q|
$$

The convergence of the suite to the value $\omega$ now follows at once. If $p_{n} / q_{n}$ is written as the general term of the suite, (34) shows that $\left|q_{n}\right|$ approaches infinity as $n$ increases without limit. The convergence then follows from (32).

In order to examine more closely into the relation between $Q$ and $q$ let us find the equation of the sphere $M$. Any base is the transform of the base of the fundamental pentahedron, $z \bar{z}+\zeta^{2}=1$, or $\rho^{2}=1$, by some transformation of the group, $z^{\prime}=(\alpha z+\beta) /(\gamma z+\delta)$. We get the transform at once from the first of equations (5), using the inverse transformation,

$$
1=\frac{\left(z \bar{z}+\zeta^{2}\right) \delta \bar{\delta}-z \delta \bar{\beta}-\bar{z} \bar{\delta} \beta+\beta \bar{\beta}}{\left(z \bar{z}+\zeta^{2}\right) \gamma \bar{\gamma}-z \gamma \bar{\alpha}-\bar{z} \bar{\gamma} \alpha+\alpha \bar{\alpha}} .
$$

This can be written in the following form:

$$
\left(z-\frac{\beta \bar{\delta}-\alpha \bar{\gamma}}{\delta \bar{\delta}-\gamma \bar{\gamma}}\right)\left(\bar{z}-\frac{\bar{\beta} \delta-\bar{\alpha} \gamma}{\delta \bar{\delta}-\gamma \bar{\gamma}}\right)+\zeta^{2}=\frac{1}{(\delta \bar{\delta}-\gamma \bar{\gamma})^{2}} .
$$

We see that this sphere has its center at the point $z=(\beta \bar{\delta}-\alpha \bar{\gamma}) /(\delta \bar{\delta}-\gamma \bar{\gamma})$ and that its radius is $1 /|\delta \bar{\delta}-\gamma \bar{\gamma}|$.

Now the transformation carrying the base of the fundamental pentahedron into the base common to two pentahedra with peaks at $p / q$ and $P / Q$ is $z^{\prime}=(P z-p) /(Q z-q)$. Hence we find that the center of $M$ is

$$
z=(P \bar{Q}-p \bar{q}) /(Q \bar{Q}-q \bar{q})
$$

and its radius is $1 /(Q \bar{Q}-q \bar{q})$. This may be stated as follows:

If $R$ is the radius of the sphere forming the common base between pentahedra with peaks at two successive fractions of Hermite, $p / q$ and $P / Q, Q$ and $q$ satisfy the equation

$$
Q \bar{Q}=q \bar{q}+1 / R \text {. }
$$

Representing the successive fractions in the suite by $p_{0} / q_{0}, p_{1} / q_{1}, \cdots$, $p_{n} / q_{n}, \cdots$, we shall now prove the following:

Writing $q_{n+1} q_{n+1}=-q_{n} q_{n}+N_{n}$, then

$$
\operatorname{Lim}_{n \rightarrow \infty} N_{n}=\infty \text {. }
$$

To prove this it is sufficient, since $N_{n}$ is an integer, to establish that there 
are only a finite number of values of $n$ yielding a given value $N$ of $N_{n}$; that is, that the line $z=\omega$ can pass through only a finite number of spheres of the system forming bases whose radii are $1 / N$. The point $\omega$ is within all these spheres, hence the spheres intersect. A reference to the fundamental pentahedron shows that two spheres of the system dividing the half space into pentahedra intersect always at an angle $\pi / 3$ or $\pi / 2$. The equators of the spheres in the $z$-plane are equal circles mutually intersecting at these angles, and enclosing the point $\omega$. Obviously only a finite number of such circles exist, and the theorem is established. [A simple geometrical construction shows that not more than four equal circles enclosing a point and mutually intersecting at one or other of the given angles exist; hence not more than four values of $N_{n}$ are equal.]

Comparison of $P$ and $p$. If we make the transformation $z^{\prime}=1 / z$ (which belongs to the group, for in writing it $z^{\prime}=i / i z$ it has the determinant 1 ), $p / q$ and $P / Q$ are transformed respectively into $q / p$ and $Q / P$. The spheres $S(p / q)$ and $S(P / Q)$ become $S(q / p)$ and $S(Q / P)$ respectively. These latter spheres are tangent to and inverse with respect to $M^{\prime}$, the transform of $M$, one lying within $M^{\prime}$ and the other without.

The transformation $z^{\prime}=1 / z$ in the plane is geometrically equivalent to an inversion in the unit circle $z \bar{z}=1$ followed by a reflection in the real axis. Then the corresponding space transformation is equivalent to an inversion in the unit sphere $z \bar{z}+\zeta^{2}=1$ followed by a reflection in the plane $\eta=0$. The latter transformation has no effect upon the dimensions of the figure.

We consider three cases according as (a) the origin is without $M,(b)$ the origin is within $M$, or $(c)$ the origin lies upon the surface of $M$.

(a) If the origin is outside $M$ the inversion in the sphere $z \bar{z}+\zeta^{2}=1$, whose center is the origin, followed by the reflection mentioned above, transforms the interior and exterior of $M$ into the interior and exterior respectively of $M^{\prime}$. The sphere $S(Q / P)$ is then inside $M^{\prime}$ and $S(q / p)$ is outside, and the latter is thus the larger. Their radii then give the inequality $1 / 2 p \bar{p}>1 / 2 P \bar{P}$, or

$$
|P|>|p| .
$$

(b) The interior of a sphere enclosing the pole of inversion is transformed into the exterior of the inverse sphere; hence in this case the interior and exterior of $M$ become the exterior and interior respectively of $M^{\prime}$. The sphere $S(Q / P)$ is then outside $M^{\prime}$ and $S(q / p)$ is inside, and the former is the larger. Whence $1 / 2 P \bar{P}>1 / 2 p \bar{p}$, or

$$
|P|<|p| .
$$

Now the only sphere of the system dividing the half-space into pentahedra which has the origin on its interior is the unit sphere $z \bar{z}+\zeta^{2}=1$ itself. The 
surface of this sphere (as of any sphere of the system) is divided by the other spheres of the system into infinitely many regions. Of these regions an infinite number are bases of pentahedra and an infinite number are lateral faces. If the line $z=\omega$ intersects the sphere in one of the bases there is a fraction $P / Q$ of the suite for which $|P|<|p|$. Since the line can intersect the sphere but once there can not be more than one such fraction. If $|\omega| \geqq 1$ there is obviously none.

The points $P / Q$ and $p / q$ are inverse with respect to $z \bar{z}=1$, the equator of $M$. Writing the equation of the inversion $z^{\prime}=1 / \bar{z}$ we have $P / Q=\bar{q} / \bar{p}$. As the fractions in this equation are in their lowest terms $P=\epsilon \bar{q}, Q=\epsilon \bar{p}$, where $\epsilon= \pm 1$ or $\pm i$. The relation $P q-p Q=-1$ gives at once the following results:

$$
P=\bar{q}, \quad Q=\bar{p}, \quad P \bar{P}=p \bar{p}-1, \quad Q \bar{Q}=q \bar{q}+1 .
$$

We shall now show that this exceptional fraction can be as far from the beginning of the suite as is desired.

Given an integer $n$ however large, a number $\omega$ can be found such that in its hermitian suite, $p_{0} / q_{0}, p_{1} / q_{1}, \cdots,\left|p_{m+1}\right|<\left|p_{m}\right|$, where $m$ is some integer greater than $n$.

Let $\omega^{\prime}$ be an irrational number on the circumference of the circle $z \bar{z}=1$, and let $p_{0} / q_{0}, \cdots, p_{n} / q_{n}, \cdots$ be its suite of Hermite, which contains an infinite number of fractions. The line $z=\omega^{\prime}$ intersects only pentahedra outside the sphere $z \bar{z}+\zeta^{2}=1$. Now $\omega^{\prime}$ can be varied slightly in any direction without altering the fact that the line $z=\omega^{\prime}$ intersects pentahedra with peaks at $p_{0} / q_{0}, \cdots, p_{n} / q_{n}$ (a finite number). The bases lying on the sphere $z \bar{z}+\zeta^{2}=1$ cluster in infinite number about each point of the equator. If we put $\omega^{\prime}=\omega$, where $\omega$ is within the limits of variation allowable and where the line $z=\omega$ intersects one of these bases, the suite for $\omega$ has an exceptional fraction for which $\left|p_{m+1}\right|<\left|p_{m}\right|$. As the pentahedra with peaks at $p_{m+1} / q_{m+1}$ lie within the sphere, this fraction is subsequent to $p_{n} / q_{n}$, whose pentahedra lie without, and the theorem is established.*

(c) If $M$ passes through the origin, $M^{\prime}$ is a plane. The sphere $S(Q / P)$ is then the reflection of $S(q / p)$ in this plane, and the two spheres are equal. Whence $1 / 2 P \bar{P}=1 / 2 p \bar{p}$, or

$$
|P|=|p| \text {. }
$$

There are infinitely many spheres of the system passing through the origin. They are the spheres which result from inverting the planes of the system $\xi=m+\frac{1}{2}, \eta=n+\frac{1}{2},(m, n=0 \pm 1, \pm 2, \cdots)$, in the sphere $z \bar{z}+\zeta^{2}=1$. The line $z=\omega$ can be chosen to intersect as many of these spheres as is desired, but we shall see that the number that can be intersected in bases is limited.

* This result is in striking contrast with that in the case of reals, where such a fraction can appear only as the second fraction of a hermitian suite-thus $1 / 0,0 / 1, \cdots$.

Trans. Am. Math. Soc. 
It will be easier to consider the planes into which these spheres are transformed. By the transformation $z^{\prime}=1 / z$ the half-line $z=\omega$ is carried into a semicircle through the origin and the point $1 / \omega$. By taking $1 / \omega$ sufficiently large the semicircle can be made to intersect as many of the planes as is desired. But the portions of these planes outside the unit spheres of the system are lateral faces of pentahedra with peaks at $\infty$, hence the bases lying in these planes are all within these unit spheres. Each of the unit spheres, which has its center always at a complex integer, intersects four planes of the system.

Now if $1 / \omega$ lies within one of the four unit spheres passing through the origin the entire semicircle lies within that sphere and can intersect only the four planes intersected by that sphere; and, since it can intersect each plane but once, the number of plane bases traversed is not more than four. If on the other hand $1 / \omega$ lies outside one of the four unit spheres through the origin the semicircle enters the fundamental pentahedron by its base before intersecting any of the planes. The semicircle then crosses a number of plane lateral faces of pentahedra and eventually passes out of the pentahedra with peaks at $\infty$ by the base of one of them. It can then intersect only the planes lying in the sphere forming that base, which are four in number. We have then the rough result that not more than four plane bases can be intersected by the semicircle; hence, the number of terms for which $|P|=|p|$ is not greater than four.*

The following two examples illustrate the three cases just discussed.

Suite for $\omega=\frac{5}{2} \frac{5}{3}$ :

$$
\frac{1}{0}, \frac{0}{1}, \frac{-1}{-4}, \frac{-1}{-5}, \frac{2}{9}, \frac{5}{23}
$$

Suite for $\omega=\frac{450+30 i}{451}$ :

$$
\frac{1}{0}, \frac{1}{1}, \frac{-1+15 i}{15 i}, \frac{-15 i}{-1-15 i}, \frac{1-30 i}{-1-30 i}, \frac{450+30 i}{451}
$$

Value of the fractions as approximations to $\omega$. It is easy to show that the distance of the approximating fraction from the limit $\omega$ tends steadily to zero; that is, that each fraction of the suite is nearer $\omega$ than the preceding fraction. For we have noted the fact that $P / Q$ and $p / q$ are inverse points with respect to the circle in which the sphere $M$ cuts the $z$-plane. The point $P / Q$ lies inside the circle, and is then nearer to any interior point of the circle than is $p / q$. Since $\omega$ is within the circle, $P / Q$ is nearer $\omega$ than is $p / q$.

This fact follows also from the following important theorem due to Hermite:

* It is easy to show that this number is not greater than three, but we omit the proof of this. We can show that one of these fractions can be as far from the beginning of the suite as desired, but that any others must remain near the beginning. 
THEOREM. If $p / q$ is a fraction in the suite for $\omega$ and $r / s$ is any other fraction for which $|s| \leqq|q|$, then

$$
\left|\omega-\frac{p}{q}\right|<\left|\omega-\frac{r}{s}\right| \cdot\left|\frac{s}{q}\right| \leqq\left|\omega-\frac{r}{s}\right| .
$$

This theorem is susceptible of a geometric proof, but it lacks the simplicity of the following proof given in Hermite's memoir: Since $x=p, y=q$ is the minimum of the form (1) of the Introduction for some value $k_{1}$ of $k$, we have

$$
(p-\omega q)(\bar{p}-\bar{\omega} \bar{q})+k_{1}^{2} q \bar{q}<(r-\omega s)(\bar{r}-\bar{\omega} \bar{s})+k_{1}^{2} s \bar{s} .
$$

This inequality holds for all integral values of $r$ and $s$ different from $p$ and $q$. If now $|s| \leqq|q|, k_{1}^{2} q \bar{q} \geqq k_{1}^{2} s \bar{s}$, and therefore

$$
(p-\omega q)(\bar{p}-\bar{\omega} \bar{q})<(r-\omega s)(\bar{r}-\bar{\omega} \bar{s})
$$

which yields at once the inequality (42).

There is thus no better rational approximation to $\omega$ than a given fraction of Hermite without having a larger denominator. Since $|q|<|Q|$ this shows that $P / Q$ is a better approximation than $p / q$.

The fraction preceding $p / q$. Let us consider all numbers whose suite of Hermite contains the fraction $p / q$, and let us fix our attention upon the fraction immediately preceding $p / q$ in each suite. How many different fractions appear? The discussion of this problem sheds an interesting light upon the geometry of the pentahedral division of the half-space. Since the line $z=\omega$ enters a pentahedron with peak at $p / q$ from a preceding pentahedron by the common base, the number of different fractions is equal to the number of the bases of pentahedra with peaks at $p / q$ which are turned upward. It is also easily shown to be the number of bases whose points of tangency with the sphere $S(p / q)$ lie above the horizontal plane, $\zeta=1 / 2 q \bar{q}$, through the center of that sphere.

Let $p_{0}, q_{0}$ be integers satisfying the equation $p q_{0}-p_{0} q=-1$. Then the transformation of the Group of Picard

$$
z^{\prime}=\frac{p z-p_{0}}{q z-q_{0}}
$$

transforms the pentahedra with peaks at $\infty$ into those with peaks at $p / q$. The pentahedra with bases in common with the preceding have their peaks at the complex integers. Hence, on transforming, the pentahedra with bases in common with those with peaks at $p / q$ have their peaks at $\left(p s-p_{0}\right) /$ $\left(q s-q_{0}\right)$, where $s$ is given all complex integral values. One of these fractions can precede $p / q$ if and only if it has a smaller denominator; that is, if

$$
\left|q s-q_{0}\right|<|q|, \quad \text { or } \quad\left|s-q_{0} / q\right|<1 \text {. }
$$

This leads to the following 
Theorem. Let $p_{0}, q_{0}$ be integers such that $p q_{0}-p_{0} q=-1$. Then the number of different fractions that can immediately precede $p / q$ in a suite of Hermite is equal to the number of complex integers enclosed by a unit circle in the $z$-plane whose center is $q_{0} / q$.

If $s_{1}, s_{2}, \cdots$ are the values of the complex integers within this circle, then the fractions which can immediately precede $p / q$ are

$$
\frac{p s_{1}-p_{0}}{q s_{1}-q_{0}}, \quad \frac{p s_{2}-p_{0}}{q s_{2}-q_{0}}, \quad \cdots .
$$

In Figure 3 a unit square with vertices at complex integers is divided into regions by unit circles with centers at the vertices. A number has been

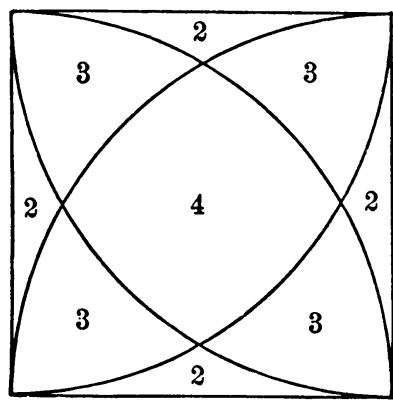

FIg. 3 affixed to each region such that if $q_{0} / q$ lies within the region the number indicates the number of complex integers lying in the unit circle with $q_{0} / q$ as center. If $q_{0} / q$ lies at a vertex of the square there is but one integer within the circle (in this case, since $q_{0} / q$ is an integer, $|q|=1$, and $p / q$ is an integer), otherwise there are two, three, or four.

If $p$ and $q$ are real, $p_{0}$ and $q_{0}$ can be chosen to be real, and $q_{0} / q$ lies upon the side of the square of the figure. We observe that in this case the number of integers enclosed is in general two, there being thus two pentahedra with bases turned upward. Now the plane $\eta=0$ cuts the pentahedra with peaks on the real axis in a configuration which is precisely the classic modular division of the half-plane. We can then make the following statement:

In the modular division of the half-plane two and only two triangles with their peaks at a point $p / q$ have their bases turned upward, unless $p / q$ is an integer, when there is but one.

Pentahedra with plane bases. Of many other geometrical considerations amenable to the methods of the preceding paragraphs* we shall treat oneto determine the number of bases of the pentahedra with peaks at $p / q$ which are plane. The condition that the base common to pentahedra with peaks at $p / q$ and $p^{\prime} / q^{\prime}$ be plane is that $S(p / q)$ and $S\left(p^{\prime} / q^{\prime}\right)$ be equal in magnitude, for these spheres are inverses with respect to the common base. Since $q^{\prime}$ is of the form $q s-q_{0}$, this requires that $\left|q s-q_{0}\right|=|q|$, or $\left|s-q_{0} / q\right|=1$. That is, $s$ is a complex integer on the circumference of the unit circle whose center is $q_{0} / q$.

* We can show, for example, that the number of bases whose points of tangency with $S(p / q)$ lie above a plane $\zeta=h$ is equal to the number of complex integers lying within a circle whose center is $q_{0} / q$ and whose radius is a simple function of $h$. Also, the number of vertices above that plane is equal to the number of mid-points of squares such as Fig. 3 within a suitable circle with $q_{0} / q$ as center. 
Referring to Figure 3 we see that if $q_{0} / q$ is an integer (in which case $p / q$ is an integer) there are four integers on the circumference. There are no points of the figure on three circles. There are points lying on two circles, but we find on solving that these points are irrational, and hence are impossible values for $q_{0} / q$. There are, however, infinitely many rational points on one circle. Whence, of the bases of pentahedra with peaks at $p / q$, four are plane if $p / q$ is a complex integer, otherwise not more than one can be plane.

8. Condition that $p / q$ be a fraction in the suite for $\omega$. We propose in this section to find necessary and sufficient conditions that a given fraction should belong to the hermitian suite of a given number without the necessity of calculating the suite. That is, we shall find the condition that the line $z=\omega$ should intersect pentahedra with peaks at $p / q$. If the line does not intersect the sphere $\Sigma(p / q)$ within which the pentahedra lie, $p / q$ is not a fraction of the suite. If the line intersects $S(p / q)$, which lies entirely within the pentahedra in question, $p / q$ certainly is a fraction of the suite. These facts give the following statement:

A necessary condition that $p / q$ belong to the suite for $\omega$ is that

a sufficient condition is that

$$
\left|\omega-\frac{p}{q}\right| \leqq \frac{1}{\sqrt{2} q \bar{q}} ;
$$

$$
\left|\omega-\frac{p}{q}\right|<\frac{1}{2 q \bar{q}} .
$$

The case in which $z=\omega$ intersects $\Sigma(p / q)$ but does not intersect $S(p / q)$ remains in doubt and requires a test of much greater nicety. If $p / q$ is a fraction of the suite the line $z=\omega$ must pass through one of the bases of the pentahedra which are turned upward. If these bases are projected orthogonally upon the $z$-plane $\omega$ must lie in the resulting figure. Now the boundaries of these bases are circles orthogonal to the $z$-plane, and they consequently project into straight lines. The upturned bases then project into a rectilinear polygon. This polygon can be constructed when we know the projections, that is, the $z$-coördinates, of the vertices of the bases.

We see from the preceding section that these upturned bases are the transforms of the bases of pentahedra with peaks at $\infty$ which lie above the complex integers $s_{1}, s_{2}, \cdots$ which lie within the unit circle with center at $q_{0} / q$, where the transformation to be applied is $z^{\prime}=\left(p z-p_{0}\right) /\left(q z-q_{0}\right)$. Now the $z$-coördinates of the vertices of the base above the integer $s$ are the $z$-coördinates of the vertices of a unit square whose center is $s$ and whose sides are parallel to the real and imaginary axes, and the $\zeta$-coördinate is $\frac{1}{2} \sqrt{2}$. Using the second of equations (5) and putting $z=z_{i}, \zeta=\frac{1}{2} \sqrt{2}$, we get on simplifying

$$
z_{i}^{\prime}=\frac{p}{q}-\frac{2\left(\bar{z}_{i}-\bar{q}_{0} / \bar{q}\right)}{q^{2}\left[2\left(z_{2}-q_{0} / q\right)\left(\bar{z}_{i}-\bar{q}_{0} / \bar{q}\right)+1\right]} .
$$


We can now state the following

Theorem. Let $p_{0}, q_{0}$ be integers such that $p q_{0}-p_{0} q=-1$, and let $s_{1}$, $s_{2}, \cdots$ be the complex integers lying within the unit circle whose center is $q_{0} / q$. Let unit squares with sides parallel to the real and imaginary axes and with centers at $s_{1}, s_{2}, \cdots$ be constructed. As we pass around the perimeter of the connected figure thus formed, let $z_{1}, z_{2}, \cdots, z_{n}$ be the $z$-coördinates of the successive vertices encountered.

From the values $z_{1}, \cdots, z_{n}$ determine $z_{1}^{\prime}, \cdots, z_{n}^{\prime}$ by means of equation (44), and form a rectilinear polygon in the z-plane by joining $z_{1}^{\prime} z_{2}^{\prime}, z_{2}^{\prime} z_{3}^{\prime}, \cdots, z_{n}^{\prime} z_{1}^{\prime}$.

Then the necessary and sufficient condition that $p / q$ be a fraction in the suite for $\omega$ is that $\omega$ lie within or upon the boundary of this polygon.

We see from the inequalities at the beginning of this section that this polygon will lie within the circle $|z-p / q|=1 / \sqrt{2} q \bar{q}$, and that it will enclose the circle $|z-p / q|=1 / 2 q \bar{q}$.* $^{*}$

The condition that $p_{0} / q_{0}$ and $p / q$, where $p q_{0}-p_{0} q=-1$, are successive fractions in the suite for $\omega$, is that the line $z=\omega$ should intersect the base common to the two pentahedra with peaks at $p_{0} / q_{0}$ and $p / q$. This base projects into a quadrilateral in the $z$-plane. Furthermore this base is derived from the base of the fundamental pentahedron by means of the transformation $z^{\prime}=\left(p z-p_{0}\right) /\left(q z-q_{0}\right)$. The $z$-coördinates of the vertices of the fundamental pentahedron are $( \pm 1 \pm i) / 2$. From these four values we get $z_{1}^{\prime}, z_{2}^{\prime}, z_{3}^{\prime}, z_{4}^{\prime}$ from (44). Then the necessary and sufficient condition that $p_{0} / q_{0}$ and $p / q$ be successive fractions in the suite for $\omega$ is that $\omega$ lie within or on the boundary of the quadrilateral $z_{1}^{\prime} z_{2}^{\prime} z_{3}^{\prime} z_{4}^{\prime}$.

\section{PART II}

\section{Regular CONTINUED FRACTIONS}

9. Preliminary account. The continued fraction

or as commonly written

$$
b_{0} \pm \frac{\epsilon_{1}}{b_{1} \pm \frac{\epsilon_{2}}{b_{2} \pm \frac{\epsilon_{3}}{b_{3} \pm}} \cdots,}
$$

$$
b_{0} \pm \frac{\epsilon_{1} \mid}{\mid b_{1} \pm} \frac{\epsilon_{2} \mid}{\mid b_{2} \pm} \frac{\epsilon_{3} \mid}{\mid b_{3} \pm} \cdots,
$$

* If $z_{k}^{\prime}$ is the vertex of this polygon which is farthest from $p / q$, we have, when $p / q$ is a fraction in the suite for $\omega$,

$$
\left|\omega-\frac{p}{q}\right| \leqq\left|z_{k}^{\prime}-\frac{p}{q}\right| \text {. }
$$

This is generally a stronger inequality than (32). I find that $\left|z_{k}^{\prime}-p / q\right|$ can be as small as but never smaller than $2 / 3 q \bar{q}$. 
will be called regular if $\epsilon_{n}= \pm 1$ or $\pm i$ and $b_{n}$ is a complex integer. ${ }^{*}$ It is clear that by multiplying each $\epsilon_{n}$ and the corresponding denominator by a suitable one of the numbers \pm 1 or $\pm i$ the continued fraction can be put in the following form:

$$
s_{0}-\frac{1 \mid}{\mid s_{1}-} \frac{1 \mid}{\mid s_{2}-} \frac{1 \mid}{\mid s_{3}-} \cdots,
$$

where $s_{n}\left(= \pm b_{n}\right.$ or $\left.\pm i b_{n}\right)$ is a complex integer, and it is this form that we shall constantly employ. $\dagger$

The value of the fraction when $\frac{1}{\mid s_{n}}-\frac{1}{\mid s_{n+1}}-\cdots$ is set equal to zero is called the $n t h$ convergent, and will be represented by $p_{n} / q_{n}$. If the fraction terminates with $s_{n}$ its value is $p_{n+1} / q_{n+1}$. If it does not terminate it is said to converge if $p_{n} / q_{n}$ approaches a limit as $n$ becomes infinite; and the value or sum of the fraction is defined to be that limit.

The first convergent $p_{1} / q_{1}$ is $s_{0}$, the second $p_{2} / q_{2}$ is $\left(s_{0} s_{1}-1\right) / s_{1}$. Putting $p_{1}=s_{0}, q_{1}=1, p_{2}=s_{0} s_{1}-1, q_{2}=s_{1}$, we note that $p_{2} q_{1}-p_{1} q_{2}=-1$. Proceeding from these two convergents, all succeeding convergents can be calculated by means of the recurrence formulæ

$$
p_{n+1}=p_{n} s_{n}-p_{n-1}, \quad q_{n+1}=q_{n} s_{n}-q_{n-1},
$$

and the relation

$$
p_{n+1} q_{n}-p_{n} q_{n+1}=-1
$$

always holds. We note that the convergents are all rational fractions in their lowest terms.

If we put $p_{0}=1, q_{0}=0$, we observe that the formulæ (46) for $n=1$ give $p_{2}$ and $q_{2}$. We shall then adjoin to the convergents defined above the convergent $p_{0} / q_{0}=1 / 0$.

The continued fraction of the suite of Hermite. We can set up at once a regular continued fraction whose convergents are the fractions of a suite of Hermite. The first two fractions of the suite we found to be $1 / 0$ and $a / 1$. In deter-

* The continued fractions involving complex integers have been little studied. Only one kind of such fraction has, so far as I know, appeared in the literature. See Hurwitz, A c t a Mathematica, vol. 11 (1887), pp. 187-200; Auric, Journal de mathé mat i q u e s, 5th ser., vol. 8 (1902), pp. 387-431.

$\dagger$ The fraction with positive signs

$$
b_{0}+\frac{1 \mid}{\mid b_{1}+}-\frac{1 !}{\mid b_{2}+}-\frac{1 \mid}{\mid b_{3}+} \cdots
$$

can be put in the form (45) as follows:

$$
b_{0}-\frac{1 \mid}{\left|-b_{1}-\right| b_{2}-} \frac{1 \mid}{\mid-b_{3}-} \cdots,
$$

that is, by merely changing the signs of $b_{1}, b_{3}, b_{5}, \cdots$. 
mining a fraction $P / Q$ in terms of the two preceding, $p^{\prime} / q^{\prime}$ and $p / q$, an integer $s$ was found and the formulæ (30) used:

$$
P=p s-p^{\prime}, \quad Q=q s-q^{\prime} . .
$$

These formulæ are identical with (46). Hence, if we put $s_{0}=a$ and let $s_{1}, s_{2}, s_{3}, \cdots$ be the integers found at each step of the calculation of the fractions, the fractions of the suite are identical with the convergents of the fraction (45).

10. A geometrical interpretation. By means of the $S$-spheres (Section 4) we can give the continued fraction a geometrical interpretation. Equation (47) shows that $S\left(p_{n+1} / q_{n+1}\right)$ and $S\left(p_{n} / q_{n}\right)$ are tangent. The convergents of a regular continued fraction, $1 / 0, s_{0} / 1, p_{2} / q_{2}, p_{3} / q_{3}, \cdots$, determine a suite of $S$-spheres, $S(1 / 0), S\left(s_{0} / 1\right), S\left(p_{2} / q_{2}\right), \cdots$, of which the first is the plane $\zeta=1$, and such that each sphere of the suite is tangent to that which precedes it.

Conversely, if $S(1 / 0), S\left(s_{0} / 1\right), S\left(p_{2} / q_{2}\right), \cdots$ is any suite of $S$-spheres (the first sphere being $\zeta=1$ ) such that each is tangent to that which precedes it, then the points of tangency of the spheres, $1 / 0, s_{0} / 1, p_{2} / q_{2}, \cdots$, are the convergents of a regular continued fraction.

To prove the converse we note that the tangency of the $S$-spheres (see the theorem at the end of Section 4) requires that $p_{n} q_{n-1}-p_{n-1} q_{n}= \pm 1$, or $\pm i, n=1,2,3, \cdots$. Beginning with the second term of the suite we can multiply numerator and denominator by a suitable one of the numbers \pm 1 or $\pm i$ so that $p_{n} q_{n-1}-p_{n-1} q_{n}=-1$ always.

Now the transformation $z^{\prime}=\left(p_{n} z-p_{n-1}\right) /\left(q_{n} z-q_{n-1}\right)$ transforms $\zeta=1$ into $S\left(p_{n} / q_{n}\right)$; and the spheres tangent to $\zeta=1$, namely $S\left(s_{m} / 1\right)$, where $m$ takes on all complex integral values, are carried into the spheres tangent to $S\left(p_{n} / q_{n}\right)$. One of these, say $S\left(s_{n} / 1\right)$, is carried into $S\left(p_{n+1} / q_{n+1}\right)$, whence

and we can take

$$
\frac{p_{n+1}}{q_{n+1}}=\frac{p_{n} s_{n}-p_{n-1}}{q_{n} s_{n}-q_{n-1}},
$$

$$
p_{n+1}=p_{n} s_{n}-p_{n-1}, \quad q_{n+1}=q_{n} s_{n}-q_{n-1},
$$

which are precisely the formulæ (46). We can determine $s_{n}$ in this manner for each value of $n$; and these values put into (45) give a regular continued fraction whose convergents are the $z$-coördinates of the points of tangency of the suite of spheres.*

We note that if $s_{n}=0$ the recurrence formulæ give $p_{n+1} / q_{n+1}=p_{n-1} / q_{n-1}$; that is, $S\left(p_{n} / q_{n}\right)$ is preceded and followed by the same sphere. Also there is an infinite convergent as often as $S(1 / 0)$ appears in the suite of $S$-spheres.

* We can in a like manner give an interpretation by means of $\Sigma$-spheres, the suite beginning with $\Sigma(1,0)$, or $\zeta=\frac{1}{2} \sqrt{2}$, and each sphere intersecting that which prenedes it orthogonally. 
11. Second geometrical interpretation. Generating curves. We shall now introduce an interpretation which will be more useful for most purposes, an interpretation which is an immediate generalization of that given for the fractions of Hermite in Section 3. We shall treat only the case of convergent continued fractions.

THEOREM. Let a moving point trace a continuous curve from a point above the plane $\zeta=1$ to the point $z=\omega$ in the z-plane. Let this curve lie entirely in the space above the z-plane except at the point of termination, and let it intersect no base more than a finite number of times. Then the z-coördinates of the successive peaks of the pentahedra through which the point passes are the convergents in order of a regular continued fraction whose sum is $\omega$.

Conversely any regular continued fraction converging to the value $\omega$ can be generated in this way.

Since the beginning of the curve is in a pentahedron with peak at $\infty$, the first convergent is $1 / 0$ as required. Also since the moving point passes from one pentahedron into another with a different peak by passing through the common base, the condition that successive $S$-spheres be tangent is satisfied. (If the point passes through a vertex it becomes necessary to choose the next pentahedron properly in order that this be true.)

As a first step toward establishing the convergence let us show that if $P$ is any point on the curve, other than the point of termination, and $p / q$ is the $z$-coördinate of the peak of the pentahedron in which $P$ lies, there are only a finite number of convergents preceding $p / q$. The portion of the curve preceding $P$ lies above some plane $\zeta=\epsilon>0$; and it is easily seen that in any finite region above such a plane there are portions of only a finite number of pentahedra. The proof of this is as follows. The sphere $\Sigma\left(p_{n} / q_{n}\right)$ in which pentahedra with peaks at $p_{n} / q_{n}$ lie has the radius $1 / \sqrt{2} q_{n} \bar{q}_{n}$, and in any finite part of the plane there are only a finite number of fractions for which $q_{n}$ has a sufficiently small value that this sphere be large enough to intersect $\zeta=\epsilon$. Further, if the sphere is sufficiently large only a finite number of the pentahedra with peaks at $p_{n} / q_{n}$ are intersected by the plane. For a transformation of the form $z^{\prime}=\left(q^{\prime} z-p^{\prime}\right) /\left(q_{n} z-p_{n}\right)$ carries the pentahedra with peaks at $p_{n} / q_{n}$ into those with peaks at $\infty$, and carries $\zeta=\epsilon$ into a sphere of radius $1 / 2 \epsilon q_{n} \bar{q}_{n}$ tangent to the $z$-plane at $q^{\prime} / q_{n}$. This latter sphere obviously intersects only a finite number of pentahedra with peaks at $\infty$. Hence $\zeta=\epsilon$ intersects only a finite number of pentahedra with peaks at $p_{n} / q_{n}$.

We conclude from the above that there are only a finite number of bases intersected by the curve before reaching the point $P$, and by hypothesis each is intersected only a finite number of times. As each passage through a base yields a new convergent, it follows that only a finite number precede the convergent $p / q$ mentioned above. 
Secondly, we must show that as $P$ approaches the termination of the curve the corresponding convergent approaches $\omega$. It has just been pointed out that the number of fractions in a finite region-in a given neighborhood of $\omega$ for example-whose $\Sigma$-spheres have a radius exceeding any small positive quantity, $\eta$ say, is finite. Unless $\omega$ is one of these rationals we can construct a sphere $R$ with $\omega$ as center of sufficiently small radius that it intersects none of these $\Sigma$-spheres. Then, if $p / q$ is the peak of the pentahedron in which $P$ lies, $\Sigma(p / q)$ has a radius less than $\eta$, when $P$ lies within $R$. As $P$ approaches $\omega$ we can decrease the radius of $R$ and likewise let $\eta$ approach zero. Since $\Sigma(p / q)$ always contains $P$ and since the radius of $\Sigma(p / q)$ approaches zero, the point of tangency, $p / q$, approaches $\omega$.

If $\omega=p^{\prime} / q^{\prime}$, a rational, the above reasoning applies to all $\Sigma$-spheres except $\Sigma\left(p^{\prime} / q^{\prime}\right)$, but as the point of tangency of this sphere is equal to $\omega$, we have convergence in this case also.

To show that any convergent regular continued fraction can be defined in this way, we consider the suite of $S$-spheres, $S(1 / 0), S\left(s_{0} / 1\right), S\left(p_{2} / q_{2}\right)$, $\cdots$, determined by the fraction. Let $A$ be a point above the plane $\zeta=1$, and join $A$ to the point of tangency of $S(1 / 0)$ and $S\left(s_{0} / 1\right)$ by a straight line, then join this point to the point of tangency of $S\left(s_{0} / 1\right)$ and $S\left(p_{2} / q_{2}\right)$ by a straight line. Continuing this process we get a continuous curve composed of segments of straight lines. It is obvious that the convergents determined by this curve are precisely those of the continued fraction.

It remains to show that a point moving along the curve thus determined approaches $\omega$ as its terminating value. If the fraction terminates we shall join the last point of tangency to the point $\omega$, the line thus lying entirely within the last $S$-sphere, and the condition demanded is obviously satisfied.

If the fraction does not terminate its convergence requires that the denominator of the $n$th convergent $p_{n} / q_{n}$ should approach infinity as $n$ increases (excepting the case where $\omega=p^{\prime} / q^{\prime}$ and $p^{\prime} / q^{\prime}$ appears infinitely often as a convergent). Then the radius of the sphere $S\left(p_{n} / q_{n}\right)$ approaches zero and a point on the straight line segment within the sphere approaches $\omega$. (If $p^{\prime} / q^{\prime}$ appears infinitely often this reasoning applies to all spheres except $S\left(p^{\prime} / q^{\prime}\right)$. But $p^{\prime} / q^{\prime}$ can not appear twice in succession as a convergent and the segment within $S\left(p^{\prime} / q^{\prime}\right)$ joins points of tangency of decreasing spheres and this part of the curve also approaches $p^{\prime} / q^{\prime}$ or $\left.\omega.\right)$ The theorem is thus established.

12. Connection of the preceding with quadratic forms. Let the curve of the preceding section be represented in parametric form

$$
z=\phi_{1}(t)+i \phi_{2}(t), \quad \zeta=f(t)
$$

the interval of the parameter being $t_{1} \geqq t \geqq t_{2}$. We have then $f\left(t_{1}\right)>1$, $\phi_{1}\left(t_{2}\right)+i \phi_{2}\left(t_{2}\right)=\omega, f\left(t_{2}\right)=0$. By referring to Section 2, we see that the 
point on the curve whose parameter is $t$ is the representative point of the definite hermitian form

$$
\left\{x-\left[\phi_{1}(t)+i \phi_{2}(t)\right] y\right\}\left\{\bar{x}-\left[\phi_{1}(t)-i \phi_{2}(t)\right] \bar{y}\right\}+[f(t)]^{2} y \bar{y} .
$$

The proposition of the preceding section can then be put in the following form:

Theorem. Let $\left(p_{0}, q_{0}\right),\left(p_{1}, q_{1}\right), \cdots$ be the successive complex integral values of $(x, y)$ yielding the minimum of the form (49) as $t$ decreases from $t_{1}$ to $t_{2}$. Then $p_{0} / q_{0}, p_{1} / q_{1}, \cdots$ are the successive convergents of a continued fraction whose sum is $\omega$.

The suite of Hermite is an example of this, in which $\phi_{1}(t)+i \phi_{2}(t)=\omega$, a constant, and $f(t)=t$.

13. The convergent as an approximation to the sum. From a knowledge of the form of the generating curve we can usually determine the degree of approximation to the sum of the continued fraction which the convergents afford. For example

If when $t<t^{\prime}$ the generating curve lies always within the right circular cone whose vertex is $\omega$ and whose generators make an angle $\alpha$ with the z-plane, then there exists an integer $m$ such that

$$
\left|\omega-\frac{p_{n}}{q_{n}}\right|<\frac{k}{q_{n} \bar{q}_{n}}, \quad \text { when } n>m,
$$

where $k=\frac{1}{2} \sqrt{2} \cot \frac{1}{2} \alpha$.

If when $t<t^{\prime}$ the generating curve lies always within a sphere of radius a tangent to the z-plane at the point $\omega$, then there exists an integer $m$ such that

where $k=\sqrt[4]{8} \sqrt{a}$.

$$
\left|\omega-\frac{p_{n}}{q_{n}}\right|<\frac{k}{\left|q_{n}\right|}, \quad \text { when } n>m,
$$

In the first, since the curve passes through $\Sigma\left(p_{n} / q_{n}\right)$ that sphere must intersect the cone in question. The inequality is a direct result of this requirement. It evidently holds after the convergent $p_{m} / q_{m}$ corresponding to the point whose parameter is $t^{\prime}$. The inequality (51) expresses the fact that $\Sigma\left(p_{n} / q_{n}\right)$ intersects the sphere of the theorem.

The first theorem is applicable to any generating curve possessing at the point $\omega$ a tangent not lying in the z-plane, the second to those curves having simple contact with the $z$-plane at that point. The inequality satisfied by the fractions of Hermite is deduced at once from (50) in putting $\alpha=\pi / 2$. If $\omega=p / q$, a rational fraction, the fraction always terminates in the first case, and terminates in the second case if $a \leqq 1 / 2 q \bar{q}$, for then the generating curve after the point $t^{\prime}$ lies always within pentahedra with peaks at $p / q$.

THEOREM. If the generating curve possesses contact of order $\lambda$ with the $z$-plane 
at the point $\omega$, there exists a constant $k$ such that all convergents satisfy the inequality

$$
\left|\omega-\frac{p_{n}}{q_{n}}\right|<\frac{k}{\left|q_{n}\right|^{2 /(\lambda+1)}} .
$$

Representing for brevity the point on the curve by $z(t), \zeta(t)$, let $a(t)$ be defined by the equation

$$
\zeta(t)=a(t)|z(t)-\omega|^{\mu}
$$

Then $\operatorname{Lim}_{t=t_{2}} a(t)=0$, or $a \neq 0$, or $\infty$, according as $\mu$ is less than, or equal to, or greater than $\lambda+1$. From this it follows that if $a_{1}<a$, after a certain point the generating curve lies entirely within the surface of revolution

$$
\zeta=a_{1}|z-\omega|^{\lambda+1}
$$

After a certain convergent $\Sigma\left(p_{n} / q_{n}\right)$ must intersect this surface. The surface is tangent to the $z$-plane at $\omega$, and as convergents are taken closer and closer to $\omega$ the surface above them approaches more nearly a plane in form. If we replace $a_{1}$ by a still smaller value $a_{2}$, the ratio of the $\zeta$-coördinates of the two surfaces being $\zeta_{2} / \zeta_{1}=a_{2} / a_{1}<1$, we see that after a certain point in the series of convergents the highest point of the sphere $\Sigma\left(p_{n} / q_{n}\right)$, viz., $z=p_{n} / q_{n}, \zeta=\sqrt{2} / q_{n} \bar{q}_{n}$, lies always within the latter surface. From this

$$
\frac{\sqrt{2}}{q_{n} \bar{q}_{n}}>a_{2}\left|\frac{p_{n}}{q_{n}}-\omega\right|^{\lambda+1} .
$$

This is an inequality of the form (52), determining a value of $k$. We can increase $k$ sufficiently to make the inequality hold for the finite number of convergents at the beginning of the series for which the inequality just found does not hold, and the theorem is established.

It is easy to show that if the curve possesses contact of order $\lambda$ the convergents do not all satisfy an inequality of the form

$$
\left|\omega-\frac{p_{n}}{q_{n}}\right|<\frac{k}{\left|q_{n}\right|^{\mu}}, \quad \text { where } \quad \mu>\frac{2}{\lambda+1}
$$

however great $k$ may be.*

14. Formulæ for the calculation of the continued fraction. The determination of the convergents defined by a given generating curve will be a very complicated procedure. This we found to be the case (Section 5) when the

* By the use of a generating curve whose contact exceeds any finite order, such as

$$
z(t)=\omega+t, \quad \zeta(t)=e^{-1 / t^{2}}
$$

we can establish the existence of convergent continued fractions whose convergents satisfy no inequality of this form, however large $k$ and however small $\mu(>0)$ be chosen. 
generating curve is a straight line. We propose to give formulæ for a continued fraction analogous to the "modified hermitian suite" of Section 6 . For this purpose we take as the convergent following $p / q$ the z-coördinate of the peak of the pentahedron in which lies the last intersection of the curve with $\Sigma(p / q)$. Since the bases of the pentahedra with peaks at $p / q$ lie very close to $\Sigma(p / q)$ this process will give in most cases in which simple generating curves are employed the convergent immediately following $p / q$ in the original fraction. In any case we shall thus set up a continued fraction differing from the original by the omission of certain convergents, and only a finite number of successive convergents will be omitted.* We note that in this modified continued fraction each convergent appears but once.

A consideration of the curve of rectilinear segments treated in Section 11 shows that if each convergent appears only once the last intersection of the curve with $\Sigma\left(p_{n} / q_{n}\right)$ lies within $S\left(p_{n+1} / q_{n+1}\right)$ and thus gives the correct convergent following $p_{n} / q_{n}$. That is, any regular continued fraction, no two of whose convergents are equal, is the modified continued fraction of some generating curve.

Let the generating curve have the equations (48). The first convergent will be $1 / 0$; the second will be the complex integer nearest to the $z$-coördinate of the last intersection of the curve with the plane $\Sigma(1 / 0)$, or $\zeta=\frac{1}{2} \sqrt{2}$. As the parameter $t$ is supposed decreasing as we proceed along the curve, the parameter of this point of intersection is the algebraically smallest root, $t^{\prime}$, of $f(t)=\frac{1}{2} \sqrt{2}$. The serond convergent is $s_{0}$, the nearest integer to $\phi_{1}\left(t^{\prime}\right)$ $+i \phi_{2}\left(t^{\prime}\right)$. We take then $p_{0}=1, q_{0}=0, p_{1}=s_{0}, q_{1}=1$, whence $p_{1} q_{0}-p_{0} q_{1}$ $=-1$.

Now let $p_{n-1} / q_{n-1}, p_{n} / q_{n}$ be successive convergents, where

$$
p_{n} q_{n-1}-p_{n-1} q_{n}=-1 \text {. }
$$

We propose to find $p_{n+1} / q_{n+1}$. The sphere $\Sigma\left(p_{n} / q_{n}\right)$ has the equation, from (17),

$$
\left(z-\frac{p_{n}}{q_{n}}\right)\left(\bar{z}-\frac{\bar{p}_{n}}{\bar{q}_{n}}\right)+\zeta^{2}-\frac{\sqrt{2} \zeta}{q_{n} \bar{q}_{n}}=0 .
$$

Replacing $z$ and $\zeta$ by their parametric values, we find that the parameter $t_{n}$ of the last intersection of the curve with this sphere is the smallest root of the equation

$$
\begin{aligned}
{\left[\phi_{1}(t)^{2}+\phi_{2}(t)^{2}+\right.} & \left.f(t)^{2}\right] q_{n} q_{n}-\left[\phi_{1}(t)+i \phi_{2}(t)\right] \bar{p}_{n} q_{n} \\
& -\left[\phi_{1}(t)-i \phi_{2}(t)\right] p_{n} \bar{q}_{n}-\sqrt{2} f(t)+p_{n} \bar{p}_{n}=0 .
\end{aligned}
$$

* Except in the case of a fraction' having $p / q(=\omega)$ appearing infinitely often, which may be rendered terminating. 
On making the transformation $z^{\prime}=\left(q_{n-1} z-p_{n-1}\right) /\left(q_{n} z-p_{n}\right)$, the sphere $\Sigma\left(p_{n} / q_{n}\right)$ becomes the plane $\zeta=\frac{1}{2} \sqrt{2}$, and the point whose parameter is $t_{n}$ becomes a point in that plane whose $z$-coördinate is (employing the second of equations (5))

$$
\begin{gathered}
{\left[\phi_{1}\left(t_{n}\right)^{2}+\phi_{2}\left(t_{n}\right)^{2}+f\left(t_{n}\right)^{2}\right] q_{n-1} q_{n}-\left[\phi_{1}\left(t_{n}\right)+i \phi_{2}\left(t_{n}\right)\right] \bar{p}_{n} q_{n-1}} \\
\frac{-\left[\phi_{1}\left(t_{n}\right)-i \phi_{2}\left(t_{n}\right)\right] p_{n} 1 \bar{q}_{n}+p_{n-1} \bar{p}_{n}}{\left[\phi_{1}\left(t_{n}\right)^{2}+\phi_{2}\left(t_{n}\right)^{2}+f\left(t_{n}\right)^{2}\right] q_{n} \bar{q}_{n}-\left[\phi_{1}\left(t_{n}\right)+i \phi_{2}\left(t_{n}\right)\right] \bar{p}_{n} q_{n}} \\
-\left[\phi_{1}\left(t_{n}\right)-i \phi_{2}\left(t_{n}\right)\right] p_{n} \bar{q}_{n}+p_{n} \bar{p}_{n}
\end{gathered}
$$

By performing a division and using the fact that $t_{n}$ is a root of (54) this can be written in the form

$$
\frac{q_{n-1}}{q_{n}}-\frac{\bar{q}_{n}\left[\phi_{1}\left(t_{n}\right)-i \phi_{2}\left(t_{n}\right)\right]-\bar{p}_{n}}{\sqrt{2} q_{n} f\left(t_{n}\right)} .
$$

The peak of the pentahedron in which this point in the plane $\zeta=\frac{1}{2} \sqrt{2}$ lies is at $s_{n}$, the nearest integer to (55). Transforming back by the inverse of the above transformation, $z^{\prime}=\left(p_{n} z-p_{n-1}\right) /\left(q_{n} z-q_{n-1}\right)$, we can write

$$
\begin{aligned}
p_{n+1}=p_{n} s_{n}-p_{n-1}, \quad q_{n+1}=q_{n} s_{n}-q_{n-1}, & \text { whence } \\
& p_{n+1} q_{n}-p_{n} q_{n+1}=-1 .
\end{aligned}
$$

We have then in brief the following method of procedure.

The first two convergents are $p_{0} / q_{0}, p_{1} / q_{1}$ where $p_{0}=1, q_{0}=0, p_{1}=s_{0}$, $q_{1}=1$, in which $s_{0}$ is the nearest integer to $\phi_{1}\left(t^{\prime}\right)+i \phi_{2}\left(t^{\prime}\right)$ where $t^{\prime}$ is the smallest root of $f(t)=\frac{1}{2} \sqrt{2}$ in the interval $t_{2} \leqq t \leqq t_{1}$.

Beginning with these two convergents, the following convergents are calculated by means of the recurrence formula (56) where, after $t_{n}$ has been found as the smallest root of (54) in the interval, $s_{n}$ is the complex integer nearest to the quantity (55).

The continued fraction with these convergents is then

$$
\omega=s_{0}-\frac{1 \mid}{\mid s_{1}-} \frac{1 \mid}{\mid s_{2}-} \cdots .
$$

15. An example. The preceding process will involve considerable labor unless the generating curve is a simple one, but it furnishes a method of setting up continued fractions with desired properties. If we choose a straight line or circle as generating curve there are only two intersections of the curve with $\Sigma\left(p_{n} / q_{n}\right)$ and the determination of $t_{n}$ involves only the solution of a quadratic equation.

We shall now examine a case in which the generating curve is a circle tangent to the $z$-plane,

$$
z=\omega+\frac{\sqrt{2} t}{1+t^{2}}, \quad \zeta=\frac{\sqrt{2} t^{2}}{1+t^{2}}, \quad 0 \leqq t \leqq 2 .
$$


This is of radius $\frac{1}{2} \sqrt{2}$ and lies in a plane parallel to $\eta=0$. The interval for $t$ is such that half of the circle, from its highest point to the point of tangency $\omega$, is considered.

We find that $s_{0}$ is the complex integer nearest to $\omega+\frac{1}{2} \sqrt{2}$. The equation (54) for the determination of $t_{n}$ is

$$
\left(v_{n} \bar{v}_{n}+2 q_{n} \bar{q}_{n}-2\right) t^{2}+\sqrt{2}\left(v_{n} \bar{q}_{n}+\bar{v}_{n} q_{n}\right)+v_{n} \bar{v}_{n}=0,
$$

in which $v_{n}=q_{n} \omega-p_{n}$. The roots of this are

$$
\frac{-\sqrt{2}\left(v_{n} \bar{q}_{n}+\bar{v}_{n} q_{n}\right) \pm \sqrt{2\left(v_{n} \bar{q}_{n}+\bar{v}_{n} q_{n}\right)^{2}-4 v_{n} \bar{v}_{n}\left(v_{n} \bar{v}_{n}+2 q_{n} \bar{q}_{n}-2\right)}}{2\left(v_{n} \bar{v}_{n}+2 q_{n}\right.} \cdot
$$

The denominator of this expression is easily seen to be positive, hence the smallest root is that with the negative sign of the radical.

Putting this value of $t_{n}$ into (55) and simplifying we find that $s_{n}$ is the nearest complex integer to the quantity

$$
\frac{q_{n-1}-u_{n}}{q_{n}}-\frac{u_{n}}{\bar{u}_{n}}\left[i B_{n}-\sqrt{u_{n} \bar{u}_{n}-\frac{1}{2}-B_{n}^{2}}\right],
$$

where $u_{n}=1 /\left(q_{n} \omega-p_{n}\right)$, and $i B_{n}$ is the imaginary part of $q_{n} u_{n}$.

If $\omega$ is real $p_{n}, q_{n}$, and $u_{n}$ will be real and (58) takes the particularly simple form

$$
\frac{q_{n-1}-u_{n}}{q_{n}}+\sqrt{u_{n}^{2}-\frac{1}{2}} .
$$

Following are two examples of this development:

$$
\begin{aligned}
\frac{1}{2} & =1-\frac{1 \mid}{\mid 4-}|1|-\frac{1 \mid}{\mid 4-} \frac{1 \mid}{\mid 1-} \frac{1 \mid}{\mid 4-} \cdots, \\
\frac{1+i}{3} & =1-\frac{1 \mid}{\mid 1+2 i-} \mid \frac{1 \mid}{\mid-1-i-} \frac{1 \mid}{\mid-2-} \frac{1 \mid}{\mid-1+i-} \frac{1 \mid}{\mid 1-2 i-} \frac{1 \mid}{\mid 1-} \cdots .
\end{aligned}
$$

The radius of the generating circle, $\frac{1}{2} \sqrt{2}$, is sufficiently gi eat so that if $\omega=p / q$, a rational, the generating circle is not contained within $\Sigma(p / q)$; hence no pentahedra with peaks at $p / q$ are intersected. This development, therefore, furnishes always non-terminating continued fractions.

An application of the inequality (51) shows that the convergents always satisfy the inequality

$$
\left|\omega-\frac{p_{n}}{q_{n}}\right| \leqq \frac{\sqrt{2}}{\left|q_{n}\right|} .
$$

This can be derived also from (58), for, since the expression under the radical must be positive, $u_{n} \bar{u}_{n} \geqq \frac{1}{2}$, which is the inequality just found.

The periodicity of these continued fractions will be discussed in a later section. 
16. Periodic regular continued fractions. The continued fraction (45) is called periodic if integers $m$ and $k$ exist such that $s_{n+k}=s_{n}$ whenever $n>m$. We shall write the periodic fraction in the form

$$
s_{0}-\frac{1 \mid}{\mid s_{1}-} \cdots \frac{1 \mid}{\mid s_{m}-} \frac{1 \mid}{\mid s_{*+1}-} \cdots \frac{1 \mid}{\underset{*}{\mid s_{m+k}}-},
$$

where the portion between the stars is repeated again and again.

Let us suppose that the fraction converges and that $\omega$ is its sum. Let $\omega_{0}$ represent the convergent fraction

Then

$$
\omega_{0}=s_{m+1}-\frac{1 \mid}{\mid s_{m+2}-} \cdots \frac{1 \mid}{\mid s_{m+k}-} .
$$

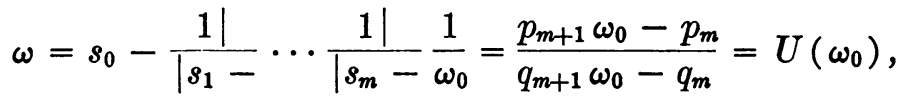

where $p_{m} / q_{m}, p_{m+1} / q_{m+1}$ are convergents as previously defined of (59). Also

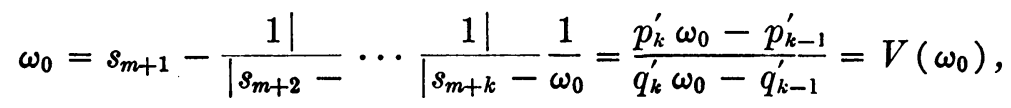

where $p_{k-1}^{\prime} / q_{k-1}^{\prime}, p_{k}^{\prime} / q_{k}^{\prime}$ are convergents of (60).

Then, employing the usual notation,

$$
\omega=U\left(\omega_{0}\right)=U V\left(\omega_{0}\right)=U V U^{-1}(\omega) .
$$

That is, the transformation $z^{\prime}=U V U^{-1}(z)$, which is of the Group of Picard since $U$ and $V$ are, carries $\omega$ into itself; $\omega$ is a fixed point of the transformation. If we write this transformation $z^{\prime}=(\alpha z+\beta) /(\gamma z+\delta)$, equation (63) exhibits the well-known result that $\omega$ is the root of a quadratic equation

$$
\gamma z^{2}+(\delta-\alpha) z-\beta=0,
$$

whose coefficients are complex integers.

We shall now establish the following important

TheoREm. The transformation $z^{\prime}=U V U^{-1}(z)$ transforms $p_{n} / q_{n}$ into $p_{n+k} / q_{n+k}$, when $n>m$.

Conversely if integers $m$ and $k$ exist such that

$$
\frac{p_{n+k}}{q_{n+k}}=T\left(\frac{p_{n}}{q_{n}}\right), \quad \text { when } \quad n>m,
$$

where $z^{\prime}=T(z)$ is a transformation of the Group of Picard, the continued fraction is periodic.

Now $p_{n} / q_{n}$ is the value of the fraction when it terminates with $s_{n-1}$. It is 
equal to the second member of $(61)$ if $\omega_{0}$ be replaced by

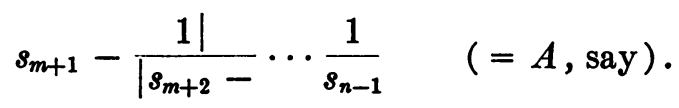

That is, $p_{n} / q_{n}=U(A)$. Similarly

$$
\frac{p_{n+k}}{q_{n+k}}=U\left(s_{m+1}-\cdots \frac{1 \mid}{\mid s_{m+k}-} \frac{1 \mid}{\mid s_{m+k+1}-} \cdots \frac{1}{s_{n+k-1}}\right)=U V(A) .
$$

Hence, $p_{n+k} / q_{n+k}=U V U^{-1}\left(p_{n} / q_{n}\right)$. This reasoning does not require the convergence of the continued fraction.

To establish the second part of the theorem, let

$$
T(z)=(\alpha z+\beta) /(\gamma z+\delta),
$$

where the integers $\alpha, \beta, \gamma, \delta$ satisfy the equation $\alpha \delta-\beta \gamma=1$. Then, if $n>m$, we have

$$
\frac{p_{n+k}}{q_{n+k}}=\frac{\alpha p_{n}+\beta q_{n}}{\gamma p_{n}+\delta q_{n}}
$$

It is easily shown that both fractions are in their lowest terms. Hence

$$
p_{n+k}=\epsilon_{n}\left(\alpha p_{n}+\beta q_{n}\right), \quad q_{n+k}=\epsilon_{n}\left(\gamma p_{n}+\delta q_{n}\right), \quad \epsilon_{n}= \pm 1, \pm i .
$$

From this and the similar equations for $p_{n+k-1}, q_{n+k-1}$, we have

$$
p_{n+k} q_{n+k-1}-p_{n+k-1} q_{n+k}=\epsilon_{n} \epsilon_{n-1}\left(p_{n} q_{n-1}-p_{n-1} q_{n}\right) \text {. }
$$

Since the first member, and also the bracketed part of the second member are each -1 , we have $\epsilon_{n} \epsilon_{n-1}=1$. Likewise $\epsilon_{n+1} \epsilon_{n}=1$, etc., whence $\epsilon_{m+1}$, $\epsilon_{m+2}, \cdots$ are either all real or all imaginary.

Finally,

$$
\begin{aligned}
p_{n+k+1} & =\epsilon_{n+1}\left(\alpha p_{n+1}+\beta q_{n+1}\right)=\epsilon_{n+1}\left[\alpha\left(p_{n} s_{n}-p_{n-1}\right)+\beta\left(q_{n} s_{n}-q_{n-1}\right)\right] \\
& =\epsilon_{n+1}\left[\left(\alpha p_{n}+\beta q_{n}\right) s_{n}-\left(\alpha p_{n-1}+\beta q_{n-1}\right)\right]=\epsilon_{n+1}\left[\frac{p_{n+k} s_{n}}{\epsilon_{n}}-\frac{p_{n+k-1}}{\epsilon_{n-1}}\right] \\
& =\epsilon_{n+1} \epsilon_{n}\left[\frac{p_{n+k} s_{n}}{\epsilon_{n}^{2}}-\frac{p_{n+k-1}}{\epsilon_{n} \epsilon_{n-1}}\right]=p_{n+k} \frac{s_{n}}{\epsilon_{n}^{2}}-p_{n+k-1} .
\end{aligned}
$$

Similarly

$$
q_{n+k+1}=q_{n+k} \frac{s_{n}}{\epsilon_{n}^{2}}-q_{n+k-1} .
$$

Hence $s_{n+k}=s_{n} / \epsilon_{n}^{2}$. If $\epsilon_{n}= \pm 1$ always, then $s_{n+k}=s_{n}$; if $\epsilon_{n}= \pm i$ always, then $s_{n+k}=-s_{n}$. In the latter case we have however $s_{n+2 k}=-s_{n+k}=s_{n}$. We thus have periodicity in all cases.

17. The character of the transformation $T$ and the resulting properties of the continued fraction. A more intimate knowledge of the periodic fraction 
necessitates a few remarks on the different types of linear transformations. Consider a transformation $z^{\prime}=(\alpha z+\beta) /(\gamma z+\delta)$ where $\alpha \delta-\beta \gamma=1$. If $\alpha+\delta$ is not real the transformation is called loxodromic. The transformations of which $\alpha+\delta$ is real are of three kinds: elliptic if $|\alpha+\delta|<2$, hyperbolic if $|\alpha+\delta|>2$, and parabolic if $|\alpha+\delta|=2$.

The fixed points of the transformation are the roots of equation (64). There is but one fixed point in the case of the parabolic transformation, in all others there are two. We shall treat first those transformations with two fixed points, $\tau_{1}$ and $\tau_{2}$. Let $C$ be the semicircle orthogonal to the $z$-plane through the two fixed points and lying in the upper half-space.

If the transformation is elliptic it is equivalent to successive inversions in two spheres passing through $C{ }^{*}$ If we call the diedral angle between these spheres $\theta / 2$ it is necessary that $\theta$ be a submultiple of $2 \pi$ in order that the group be discontinuous. The transform of any sphere through $C$ is a sphere through $C$ making an angle $\theta$ with the original. After a finite number of applications of the transformation all points of space are brought back to their original positions.

If $T$ is elliptic there exists an integer $\nu$ such that $T^{\nu}=1$. Then

$$
p_{n+k_{\nu}} / q_{n+k_{\nu}}=p_{n} / q_{n}
$$

when $n>m$. The convergents $p_{m+1} / q_{m+1}, \cdots, p_{m+k_{\nu}} / q_{m+k_{\nu}}$ appear infinitely many times, and the continued fraction does not converge. We shall lay this case aside.

If $T$ is hyperbolic it is equivalent to successive inversions in two spheres with centers in the $z$-plane and cutting $C$ orthogonally. Each circle $C^{\prime}$ through $\tau_{1}$ and $\tau_{2}$ is transformed into itself. By repetitions of $T$ the transforms of all points of space (one fixed point excepted) approach one of the fixed points ( $=\omega$, the sum of the fraction), all the transforms of a point lying on the circie $C^{\prime}$ through that point.

Let circles $C_{1}^{\prime}, C_{2}^{\prime}, \cdots, C_{k}^{\prime}$ be drawn through the fixed points in such a manner that $C_{r}^{\prime}$ intersects $\Sigma\left(p_{m+r} / q_{m+r}\right)$. Now $\Sigma\left(p_{n} / q_{n}\right), n>m$, is merely the transform by applications of $T$ of one of these $k \Sigma$-spheres, hence the $k$ circles intersect all $\Sigma$-spheres after $\Sigma\left(p_{m} / q_{m}\right)$. These circles make angles greater than zero with the $z$-plane, and we see readily that we can construct a right circular cone with vertex at $z=\omega$ and with axis perpendicular to the $z$-plane which intersects all the $\Sigma$-spheres of the convergents. From this we conclude by the reasoning of the first theorem of Section 13 that there exists a consiant $k$ such that all the convergents satisfy the inequality

$$
\left|\omega-\frac{p_{n}}{q_{n}}\right|<\frac{k}{q_{n} \bar{q}_{n}}
$$

\footnotetext{
* For the facts mentioned in this section see Poincaré, loc. cit.
} 
If $T$ is loxodromic it is equivalent to the successive performance of two transformations with the fixed points $\tau_{1}$ and $\tau_{2}$, one elliptic, the other hyperbolic, but the angle $\theta$ of the former need not be a submultiple of $2 \pi$. $C$ is the only fixed circle of the transformation, but we can construct a fixed surface. Let $H$ be the circle of intersection of two spheres orthogonal to $C$. Then $H$ is a fixed circle for the elliptic component of $T$. If we form a surface $\Gamma$ by constructing circles through $\tau_{1}$ and $\tau_{2}$ and the points of $H$ this surface is invariant under the transformation $T$, for the elliptic component of $T$ transforms one of these circles through a point of $H$ into another such circle, and the hyperbolic component leaves the latter circle invariant.

Surfaces $\Gamma_{1}, \cdots, \Gamma_{k}$ can be constructed to intersect $\Sigma\left(p_{m+1} / q_{m+1}\right), \cdots$, $\Sigma\left(p_{m+k} / q_{m+k}\right)$; and $\Sigma\left(p_{n} / q_{n}\right), n>m$, will always intersect one of these surfaces. Now $H_{1}, \cdots, H_{k}$ by means of which these surfaces are constructed will lie entirely in the upper half-space, and the $\Gamma$-surfaces will in the neighborhood of $\omega$ all lie within a suitable cone with vertex at $\omega$ and with axis perpendicular to the $z$-plane. This cone will intersect all $\Sigma$-spheres after a certain point in the suite of convergents, and as before it can be enlarged to intersect all $\Sigma$-spheres of the suite. Therefore, a constant $k$ exists such that all the convergents satisfy the inequality (65).

The parabolic transformation presents a very different aspect. In this case there is a single fixed point $\tau$. Any circle through $\tau$ and tangent to a certain line in the $z$-plane is transformed into itself. The transformation is equivalent to successive inversions in two spheres through $\tau$ orthogonal to the line just mentioned (and hence tangent to one another).

Let $C_{1}^{\prime}, \cdots, C_{k}^{\prime}$ be members of the family of fixed circles just mentioned such that $C_{r}^{\prime}$ intersects $\Sigma\left(p_{m+r} / q_{m+r}\right)$. Then $\Sigma\left(p_{n} / q_{n}\right), n>m$, intersects one of these circles. A sphere tangent to the $z$-plane at $z=\tau=\omega$ and enclosing all these circles can be constructed, and its radius can be enlarged sufficiently to intersect also the $\Sigma$-spheres preceding $\Sigma\left(p_{m+1} / q_{m+1}\right)$. From the reasoning of the second theorem of Section 13 a constant $k$ exists such that the convergents all satisfy the inequality

$$
\left|\omega-\frac{p_{n}}{q_{n}}\right|<\frac{k}{\left|q_{n}\right|} .
$$

It is easily shown that they do not all satisfy an inequality of the form (65) however large $k$ be chosen.

The preceding treatment covers all possible cases. If we add the remark that the fixed points of the hyperbolic and loxodromic transformations are always irrational, whereas the fixed point of the parabolic transformation is always rational, we can state the following

ThEOREM. The convergents of a convergent periodic regular continued fraction 
always satisfy an inequality of the form (66). The sum of the fraction is irrational or rational according as an inequality of the form (65) is or is not satisfied.

18. On quadratic irrationals. It is known from the theory of quadratic forms that there are infinitely many transformations of the Group of Picard leaving invariant the roots of an equation

$$
A z^{2}+B z+C=0,
$$

in which $A, B, C$ are complex integers and the discriminant, $D=B^{2}-4 A C$, is not a perfect square.* The roots are irrational. If $T$ is one such transformation all powers of $T$ also leave the roots invariant. Since there are only a finite number of elliptic transformations possible, it follows that $T$ can be chosen to be either hyperbolic or loxodromic.

For a better understanding of what follows we shall prove the following

Theorem. The necessary and sufficient condition that $T$ can be chosen to be hyperbolic is that $D \bar{D}$ be a perfect square.

The roots of the given equation being the fixed points of $T$,

$$
z^{\prime}=(\alpha z+\beta) /(\gamma z+\delta),
$$

they are the roots of the equation

$$
\gamma z^{2}+(\delta-\alpha) z-\beta=0 .
$$

If $A, B, C$ are without a common factor, as we can suppose without loss of generality, we have

$$
\gamma=u A, \quad \delta-\alpha=u B, \quad \beta=-u C,
$$

where $u$ is a complex integer. If we put $\alpha+\delta=t$, whence

$$
\delta=(t+u B) / 2, \quad \alpha=(t-u B) / 2,
$$

the relation $\alpha \delta-\beta \gamma=1$ yields the well-known equation of Pell

$$
t^{2}-D u^{2}=4 \text {. }
$$

Conversely any integral solutions of Pell's equation which yield integral values of $\alpha$ and $\delta$ provide a transformation leaving the roots of the equation unchanged.

Let $T$ be hyperbolic. Then $t$ is real, and $D u^{2}$ is real. Writing $D=m+n i$, $u=a+b i$, and equating to zero the imaginary part of $D u^{2}$, we have

$$
\left(a^{2}-b^{2}\right) n+2 b m=0 .
$$

If either $m$ or $n$ is zero, obviously $D \bar{D}$ is a perfect square. Otherwise we can

${ }^{*}$ Fricke-Klein, Theorie der automorphen F'unctionen, vol. 1, pp. 464-467. 
write this in the form

$$
(a n+b m)^{2}=b^{2}\left(m^{2}+n^{2}\right)=b^{2} D \bar{D} .
$$

We can suppose $b \neq 0$, for $b=0$ requires $a=0$, and we have $t= \pm 2$, $u=0$, yielding the transformation $z^{\prime}=z$. The equation then shows that $D \bar{D}$ is a perfect square, and the condition is necessary.

To prove it sufficient, we suppose $D \bar{D}=G^{2}$, where $G(>0)$ is an integer. The circle in the $z$-plane with the line joining the roots of (67) as a diameter has its center at the point $-B / 2 A$ and its radius is $|\sqrt{D} / 2 A|$, or $\sqrt{G / 4 A \bar{A}}$. Its equation is

$$
\left(z+\frac{B}{2 A}\right)\left(\bar{z}+\frac{\bar{B}}{2 \bar{A}}\right)-\frac{G}{4 A \bar{A}}=0 .
$$

The sphere, $R$, with this circle as equator is the representative sphere of the indefinite hermitian form

$$
(2 A x+B y)(2 \bar{A} \bar{x}+\bar{B} \bar{y})-G y \bar{y} .
$$

From the theory of these forms it is known that among the portions of this representative sphere lying in the different pentahedra a finite number can be chosen from which all others can be derived by suitable applications of transformations of the Group of Picard.*

Let $U$ be a transformation of the group leaving the roots of (67) unaltered. The circle $C$, orthogonal to the z-plane through the roots, is a fixed circle of $U$. Also $C$ lies on the surface of $R$. Let $\Pi$ be a pentahedron through which $C$ passes (we use here the single, not the double pentahedron), and let $\Pi_{1}, \Pi_{2}$, $\cdots$ be the transforms of $\Pi$ by $U, U^{2}, \cdots$. Let $B, B_{1}, B_{2}, \cdots$ be the portions of the surface of $R$ lying in $\Pi, \Pi_{1}, \Pi_{2}, \cdots$. Since only a finite number of these are non-congruent (i. e., not derivable from one another by a transformation of the group), we can choose two congruent ones $B_{m}$ and $B_{n}$. The transformation carrying $B_{m}$ into $B_{n}$ is that carrying $\Pi_{m}$ into $\Pi_{n}$, that is $U^{n-m}$. If $\theta$ is the angle of the elliptic component of $U^{n-m}$ any sphere through $C$ is transformed into another making an angle $\theta$ with it. Such a sphere is $R$, which is transformed into itself; hence $\theta=0$ or $\pi$. If $\theta=0, U^{n-m}$ has no elliptic component and is therefore hyperbolic; if $\theta=\pi, U^{2(n-m)}$ is hyperbolic. The transformation $T$ can be chosen to be this hyperbolic transformation, and the condition is thus sufficient.

If $D \bar{D}$ is a perfect square we shall call a root of the equation a hyperbolic irrational, otherwise we shall call it a loxodromic irrational.

19. Periodicity when $\omega$ is a hyperbolic irrational. We propose now to establish certain general theorems concerning periodicity when the continued

\footnotetext{
${ }^{*}$ Fricke-Klein, loc. cit., p. 467.
} 
fraction is defined by a generating curve as described in Section 11. We shall treat only curves having a continuously turning tangent in the neighborhood of the point $\omega$. For periodicity, if $\omega$ is a quadratic irrational, this tangent cannot lie in the z-plane. Let $\omega^{\prime}$ be the other root of the quadratic equation of the form (67) satisfied by $\omega$, and let $T$ be a hyperbolic transformation with $\omega, \omega^{\prime}$ as fixed points and let all points be carried to the neighborhood of $\omega$ by repeated applications of $T$.

Let $C^{\prime}$ be a circle through $\omega, \omega^{\prime}$ and tangent to the generating curve at $\omega$. Then $C^{\prime}$ is a fixed circle of $T$, and an arc $A$ of $C$ can be found such that all subsequent parts of the circle in the upper half-space are derived from this arc by applications of $T, T^{2}$, etc. Hence the subsequent pentahedra through which $C^{\prime}$ passes are derived from those through which $A$ passes by these transformations, and the same is true of their peaks.

Now suppose that $A$ does not intersect an edge adjoining a base and is not tangent to a base. Then a small circle $K$ can be drawn with a point on $A$ as center and lying in a plane perpendicular to the arc at this point such that the surface formed by circles through $\omega, \omega^{\prime}$ and the points of $K$ intersects always the same bases and in the same order as $A$. This surface being also invariant under $T$ intersects throughout its whole length the same bases and in the same order as $C^{\prime}$. In the infinitesimal neighborhood of $\omega$ this surface is a cone surrounding the tangent to the generating curve. After a certain point the generating curve lies entirely within this surface, and intersects the same pentahedra as $C^{\prime}$. The $z$-coördinates of the peaks then satisfy the relation $p_{n+k} / q_{n+k}=T\left(p_{n} / q_{n}\right)$ for a suitable value of $k$, and the fraction is periodic. Hence, the following

THEOREM. If at the hyperbolic irrational $\omega$ the circle through $\omega, \omega^{\prime}$ tangent to the generating curve does not intersect an edge bounding a base of some pentahedron and is not tangent to a base the continued fraction is periodic.

If $C^{\prime}$ intersects an edge adjoining a base the generating curve may in the neighborhood of $\omega$ intersect the transforms of any of the bases adjoining this edge. If however a surface be formed by circles through $\omega, \omega^{\prime}$ and the edge this surface will divide the cone at $\omega$ into two parts, and if the generating curve lies after a certain point always within one of these compartments it will intersect always the transforms of the same base and the fraction will be periodic. This will always occur if the generating curve is algebraic unless it lies entirely upon the algebraic surface just formed, for it can intersect that surface only a finite number of times. The case in which $C^{\prime}$ is tangent to a base can be treated in a similar manner.

The preceding theorem requires alteration for the modified continued fraction for which formulæ were given in Section 14. Here the convergent following $p / q$ does not depend upon the intersection of the curve with a base 
but with the sphere $\Sigma(p / q)$. The points of this spherical surface within the circular quadrilateral formed by the intersection of the four lateral faces of a pentahedron with peak at $p / q$ all lie within pentahedra having the same peak. If we replace the condition of the theorem by the condition that $C^{\prime}$ shall " not intersect the circle in which the lateral face of some pentahedron intersects the sphere of its vertices" the theorem holds. As before this condition can in general be dispensed with if the generating curve is algebraic.

20. Periodicity when $\omega$ is a loxodromic irrational. If $T$ is loxodromic the only fixed circle is the circle $C$ through $\omega, \omega^{\prime}$ orthogonal to the $z$-plane. An arc $A$ of $C$ can be found such that the part of the circle between $A$ and $\omega$ is derived from $A$ by the transformations $T, T^{2}$, etc. Suppose that $A$ intersects no edge bounding a base. [Since $C$ is orthogonal to the z-plane it cannot be tangent to a base.] About a point of $A$ let a small circle $H$, as in Section 17, be constructed-that is, the circle of intersection of two spheres orthogonal to $C$. The surface $\Gamma$ formed by circles through $\omega, \omega^{\prime}$ and the points of $H$ is invariant under $T$. Further, if $H$ be sufficiently small this surface intersects the same bases and in the same order as $A$, and hence the same bases as $C$ throughout its entire course. Now let $H$ be enlarged until $\Gamma$ intersects an edge or becomes tangent to a base. Call this surface $\Gamma_{0}$. In the infinitesimal neighborhood of $\omega, \Gamma_{0}$ is a right circular cone with the line $z=\omega$ as axis. We have the

Theorem. If $\omega$ is a loxodromic irrational and the circle $C$ through $\omega, \omega^{\prime}$ orthogonal to the z-plane does not intersect an edge bounding a base, there exists a right circular cone $\Gamma_{0}$ with vertex at $\omega$ and axis perpendicular to the z-plane, such that if the tangent to the generating curve lies within $\Gamma_{0}$ the fraction is periodic, and if it lies without the fraction is not periodic.

To prove the latter part of the theorem let $H$ be increased until the tangent to the generating curve is tangent to $\Gamma$ at the point $\omega$. Now $\Gamma$ will intersect certain bases not intersected by $C$. Let $B$ be one of these bases. The transforms of $B$ by $T, T^{2}, \cdots$ approach $\omega$ and owing to the elliptic component of $T$ are rotated about the circle $C$ through multiples of an angle incommensurable with $2 \pi$. Infinitely many of these are intersected by the generating curve. But no transformation $U$ exists such that each one intersected is derived from the preceding by an application of $U$. For all would then be derived from the first by $U, U^{2}, \cdots$, and since the angle of the elliptic component of $U$ must be incommensurable with $2 \pi$ certain of these bases are on the opposite side of $C$ from the generating curve and are not intersected by it. The fraction cannot then be periodic.

The theorem relative to the modified continued fraction is stated in the same terms as before-by substituting "the circle in which a lateral face of a pentahedron intersects the sphere of its vertices" for " an edge bounding a base." The surface $\Gamma_{0}$ is in general different. 
Periodicity of the continued fraction derived from the suite of Hermite. The preceding theorems establish the facts concerning the continued fraction whose convergents are the fractions of Hermite. The generating curve is algebraic and even when $C^{\prime}$ intersects an edge does not lie on the exceptional surface mentioned in Section 19. Furthermore it is perpendicular to the $z$-plane and lies always inside the cone $\Gamma_{0}$ of Section 20 when that cone exists. Hence the

THEOREM. The continued fraction whose convergents are the fractions of Hermite is always periodic when $\omega$ is a hyperbolic irrational. It is periodic when $\omega$ is a loxodromic irrational except when the circle through $\omega, \omega^{\prime}$ orthogonal to the z-plane intersects an edge adjoining a base of some pentahedron.

21. Periodicity when $\omega$ is rational. If $\omega=p / q$, a rational, we know that the fraction terminates unless the tangent to the curve at $\omega$ lies in the $z$-plane, and that it is not periodic if the curve has contact with the z-plane of higher order than the first. We shall treat then curves of simple contact. Let $C$ be the osculating circle of the curve at $\omega$. Then $C$ is tangent to the $z$-plane, but does not lie in that plane. Obviously if $C$ lies entirely within the pentahedra with peaks at $p / q$ the same will be true of the generating curve after a certain point and the fraction will terminate.

Under what circumstances is $C$ a fixed circle for some parabolic transformation with $\omega$ as fixed point? These transformations are those carrying some pentahedron with peak at $p / q$ into some other. If we make the transformation $z^{\prime}=\left(q_{0} z-p_{0}\right) /(q z-p)$, where $p q_{0}-p_{0} q=-1$, the pentahedra with peaks at $p / q$ become those with peaks at $\infty$. The circle $C$ becomes a straight line $L$ parallel to the $z$-plane. The parabolic transformations carrying the pentahedra with peaks at $\infty$ into themselves are $z^{\prime}=z+\beta$ where $\beta$ is a complex integer. The fixed lines are those parallel to the line joining the origin to the point $z=\beta$; that is, parallel to $\bar{\beta} z-\beta \bar{z}=0$, or if we like parallel to the line

$$
\bar{\beta}\left(z-q_{0} / q\right)-\beta\left(\bar{z}-\bar{q}_{0} / \bar{q}\right)=0
$$

through $q_{0} / q$, which is parallel to the preceding. The fixed lines for the transformation $z^{\prime}=z+\beta$ meet this line at $\infty$. Transforming back (putting $\left(q_{0} z-p_{0}\right) /(q z-p)$ for $z$ in this equation) we get the line

$$
\beta q^{2}(z-p / q)-\bar{\beta} \bar{q}^{2}(\bar{z}-\bar{p} / \bar{q})=0 .
$$

The parallel lines previously mentioned become circles through $p / q$ tangent to this line. Giving $\beta$ all integral values we get the lines to one of which $C$ must be tangent in order to be a fixed circle of some parabolic transformation with $p / q$ as fixed point.

The preceding line is not less general on account of the factor $q^{2}$. Putting 
$\beta=\bar{\beta}^{\prime} \bar{q}^{2}$, an integer, we have

$$
\bar{\beta}^{\prime}(z-p / q)-\beta^{\prime}(\bar{z}-\bar{p} / \bar{q})=0 .
$$

That is, the tangents to the fixed circles at $p / q$ are parallel to the lines joining the origin to all complex integers $\beta^{\prime}$. Otherwise put, it is necessary and sufficient that the slope, $d \eta / d \xi$, of the tangent to the circle be rational.

Let the slope $d \eta / d \xi$ of the tangent to the generating curve be rational. Then $C$ is a fixed circle of some transformation $T$, and we see just as in the preceding cases that the peaks of the pentahedra through which $C$ passes fall into a recurrence scheme $p_{n+k} / q_{n+k}=T\left(p_{n} / q_{n}\right)$. If $C$ intersects no edge bounding a base and is tangent to no base a circle $K$ can be constructed with a point on $C$ as center and lying in a plane perpendicular to $C$, such that the circles tangent to $C$ at the point $\omega$ and passing through the points of $K$ form a surface which intersects the same bases and in the same order as does $C$. These circles possess simple contact with $C$ whereas the generating curve has contact of a higher order, hence after a certain point the generating curve lies entirely within this surface. Its convergents then satisfy the relation stated above, and the fraction is thus periodic.

If $C$ intersects an edge or is tangent to a base we cannot infer periodicity. If however the generating curve is algebraic we have, as in the hyperbolic case, periodic fractions in general in this case also.

If the tangent to the generating curve has not a rational slope the fraction is not periodic, for the convergents can not satisfy after a certain point a relation of the form $p_{n+k} / q_{n+k}=T\left(p_{n} / q_{n}\right)$. This is easiest seen by considering the line $L$ and the transformations $z^{\prime}=z+\beta$. If the slope of $L$ is not rational the repeated application of a transformation of this form will carry any pentahedron which $L$ intersects to a distance as great as we like from $L$.

We have established then the following

THEOREM. If the generating curve has simple contact with the z-plane at the rational point $\omega$, the fraction terminates if the oscularing circle $C$ of the curve at the point $\omega$ lies within pentahedra with peaks at $\omega$. It is not periodic if the slope, $d \eta / d \xi$, of the tangent to the generating curve is irrational.

If the slope is rational the fraction is periodic unless $C$ intersects an edge adjoining a base of some pentahedron or is tangent to some base.

A similar theorem holds for the modified continued fraction with the change of phraseology previously mentioned.

The periodicity of the continued fraction of Section 15. Here the osculating circle is the generating curve itself. Its radius, $\frac{1}{2} \sqrt{2}$, is sufficiently great to prevent the termination of the fraction. The circle lies in a plane parallel to $\eta=0$, hence $d \eta / d \xi=0$, a rational. The fraction is periodic unless the 
circle meets the circle in which the lateral face of some pentahedron intersects the sphere of its vertices. This exception is a trivial one. For the generating circle likewise intersects the successive transforms of this circle on the sphere of vertices. We have thus at regular intervals in the formation of the fraction two possible values of the next convergent at our disposal. If the choice be properly made each time the fraction will be periodic. With this understanding for the exceptional case, the continued fraction is periodic for all rational values of $\omega$.

22. The number field $[1, \epsilon]$. All the results of this paper can, with suitable modifications, be extended to the number field in which an integer is defined to be a number of the form $m+n \epsilon$, where $m$ and $n$ are real integers and $\epsilon=\frac{1}{2}+\frac{1}{2} i \sqrt{3}$. Bianchi* has found the fundamental polyhedron of the group $z^{\prime}=(\alpha z+\beta) /(\gamma z+\delta), \alpha \delta-\beta \gamma=1$, where $\alpha, \beta, \gamma, \delta$ are integers of this field. Three of the polyhedra into which the half-space is divided form the heptahedron whose lateral faces are the six planes $\xi= \pm \frac{1}{2}, \xi+\eta \sqrt{3}$ $= \pm 1, \xi-\eta \sqrt{3}= \pm 1$, and whose base is the unit sphere $z \bar{z}+\zeta^{2}=1$. This heptahedron plays the rôle of the fundamental double pentahedron of the preceding pages.

The University of Edinburgh,

$$
\text { April, } 1917
$$

*Mathematische Annalen, vol. 38 (1891), pp. 313-333. 